\title{
Improved light-cone harmonic oscillator model for the pionic leading-twist distribution amplitude
}

\author{
Tao Zhong, ${ }^{1, *}$ Zhi-Hao Zhu, ${ }^{2}$ Hai-Bing Fu ${ }^{1,3, \dagger}$ Xing-Gang Wu, ${ }^{3,4, \$}$ and Tao Huang $\odot^{5, \S}$ \\ ${ }^{1}$ Department of Physics, Guizhou Minzu University, Guiyang 550025, P.R. China \\ ${ }^{2}$ College of Physics and Materials Science, Henan Normal University, Xinxiang 453007, P.R. China \\ ${ }^{3}$ Department of Physics, Chongqing University, Chongqing 401331, P.R. China \\ ${ }^{4}$ Chongqing Key Laboratory for Strongly Coupled Physics, Chongqing 401331, P.R. China \\ ${ }^{5}$ Institute of High Energy Physics and Theoretical Physics Center for Science Facilities, \\ Chinese Academy of Sciences, Beijing 100049, P.R. China
}

(Received 9 February 2021; accepted 26 June 2021; published 22 July 2021)

\begin{abstract}
In this paper, we study the pion leading-twist distribution amplitude (DA) $\phi_{2 ; \pi}(x, \mu)$ by improving the traditional light-cone harmonic oscillator model within the reconstruction of the function $\varphi_{2 ; \pi}(x)$. In order to constrain the model parameters, we calculate its moments $\left.\left\langle\xi^{n}\right\rangle_{2 ; \pi}\right|_{\mu}$ in the framework of the QCD background field theory sum rule up to tenth order. Considering the fact that the sum rule of the zeroth moment $\left.\left\langle\xi^{0}\right\rangle_{2 ; \pi}\right|_{\mu}$ cannot be normalized, we suggest a more reasonable sum rule formula for $\left.\left\langle\xi^{n}\right\rangle_{2 ; \pi}\right|_{\mu}$. Then, we obtain the values of $\left.\left\langle\xi^{n}\right\rangle_{2 ; \pi}\right|_{\mu_{0}}$ with $n=(2,4,6,8,10)$ at the initial scale $\mu_{0}=1 \mathrm{GeV}$. The first two moments are $\left.\left\langle\xi^{2}\right\rangle_{2 ; \pi}\right|_{\mu_{0}}=0.271 \pm 0.013$ and $\left.\left\langle\xi^{4}\right\rangle_{2 ; \pi}\right|_{\mu_{0}}=0.138 \pm 0.010$, and the corresponding Gegenbauer moments are $a_{2}^{2 ; \pi}\left(\mu_{0}\right)=0.206 \pm 0.038$ and $a_{4}^{2 ; \pi}\left(\mu_{0}\right)=0.047 \pm 0.011$, respectively. After fitting the moments $\left.\left\langle\xi^{n}\right\rangle_{2 ; \pi}\right|_{\mu}$, we obtain the appropriate model parameters by using the least squares method. The resultant behavior for the twist-2 pion DA is closer to the AdS/QCD and lattice results, but narrower than that obtained using the Dyson-Schwinger equation. Furthermore, we calculate the pion-photon transition form factors (TFFs) and $B \rightarrow \pi$ TFFs within the light-cone sum rule approach, which conform with experimental and theoretical results.
\end{abstract}

DOI: $10.1103 /$ PhysRevD.104.016021

\section{INTRODUCTION}

Light meson light-cone distribution amplitudes (DAs) are universal nonperturbative objects, which describe the momentum fraction distributions of partons in a meson for a particular Fock state. These DAs enter exclusive processes based on the factorization theorems in the perturbative QCD theory (pQCD), and therefore they are key parameters in the QCD predictions for corresponding processes. In the standard treatment of exclusive processes in QCD proposed by Brodsky and Lepage [1], cross sections are arranged according to different twist structures of meson DAs, in which the leading-twist DA contribution usually dominates due to the fact that the contributions

\footnotetext{
*zhongtao1219@sina.com

fuhb@cqu.edu.cn

*wuxg@cqu.edu.cn

\$huangtao@ihep.ac.cn
}

Published by the American Physical Society under the terms of the Creative Commons Attribution 4.0 International license. Further distribution of this work must maintain attribution to the author(s) and the published article's title, journal citation, and DOI. Funded by SCOAP. from the higher twists are highly power suppressed at short distance. Thereafter, the study of the pionic leading-twist DA-which describes the momentum distribution of the valence quarks in a pion-attracted much attention in the literature.

So far, a large number of studies on the pionic leadingtwist DA rely on its Gegenbauer expansion series [2,3]; the nonperturbative expansion coefficients, denoted $a_{n}^{2 ; \pi}(\mu)$, are called Gegenbauer moments which encode the longdistance dynamics at low energy scales $(\sim 1 \mathrm{GeV})$, and only the even Gegenbauer moments are nonzero due to the isospin symmetry. In many applications of the pion leading-twist DA involving a high normalization scale, the higher Gegenbauer moment contributions are suppressed due to the fact that the anomalous dimension of $a_{n}^{2 ; \pi}(\mu)$ grows with $n$, and only the lowest Gegenbauer moments are retained. Therefore, people usually adopt the truncated form involving only the first few terms in the Gegenbauer expansion series as an approximate form of $\phi_{2 ; \pi}(x, \mu)$. Those Gegenbauer moments can be calculated directly via some nonperturbative methods, such as QCD sum rules [4-9] or lattice gauge theory [10-13]. Using QCD sum rules to calculate $a_{n}^{2 ; \pi}(\mu)$ is realized by calculating $\left.\left\langle\xi^{n}\right\rangle_{2 ; \pi}\right|_{\mu}$. 
Recently, we realized that these calculations need to be improved. Using the QCD sum rules method, the analytic formula is for $\left.\left\langle\xi^{n}\right\rangle_{2 ; \pi}\right|_{\mu} \times\left.\left\langle\xi^{0}\right\rangle_{2 ; \pi}\right|_{\mu}$, but this is usually seen as the sum rules of $\left.\left\langle\xi^{n}\right\rangle_{2 ; \pi}\right|_{\mu}$ due to the normalization of $\phi_{2 ; \pi}(x, \mu)$. In fact, due to the incompleteness of our sum rules calculation, the deviation of $\left.\left\langle\xi^{0}\right\rangle_{2 ; \pi}\right|_{\mu}$ from the normalization must be considered. This motivates us to recalculate the moments of the pionic leading-twist DA with QCD sum rules.

On the other hand, the truncated form mentioned above does not seem to be enough to describe the behavior of the DA below the low energy scale. A natural idea is to consider the contributions of higherorder Gegenbauer polynomials, which requires the calculation of higher-order Gegenbauer moments. But there is a very serious difficulty in doing so, that is, it is difficult to get reliable higher $a_{n}^{2 ; \pi}(\mu)$. Through the mathematical relationship between $a_{n}^{2 ; \pi}(\mu)$ and $\left.\left\langle\xi^{n}\right\rangle_{2 ; \pi}\right|_{\mu}$, we can find that with the increase of order $n$, the reliability of $a_{n}^{2 ; \pi}(\mu)$ decreases sharply, which makes our calculation of higher $a_{n}^{2 ; \pi}(\mu)$ meaningless. So people try to study the behavior of $\phi_{2 ; \pi}(x, \mu)$ in other ways. In Ref. [14], in the framework of the Dyson-Schwinger equations, the authors obtained pionic leading-twist DAs (DS model) that are concave and significantly broader than the asymptotic DAs. Making use of the approximate bound-state solution of a hadron in terms of the quark model as the starting point, Brodsky, Huang, and Lepage (BHL) suggested the light-cone harmonic oscillator (LCHO) model which is obtained by connecting the equal-time wave function (WF) in the rest frame and the WF in the infinite-momentum frame [15]. Meanwhile, the holographic Schrödinger equation for a meson maps onto the fifth dimension of anti-de Sitter space with a QCD potential (AdS/QCD) [16].

In this paper, we study the pionic leading-twist DA $\phi_{2 ; \pi}(x, \mu)$ based on the improved LCHO model. The determination of model parameters depends on the moments $\left.\left\langle\xi^{n}\right\rangle_{2 ; \pi}\right|_{\mu}$ rather than the Gegenbauer moments $a_{n}^{2 ; \pi}(\mu)$, and we will adopt a new method, that is, the least squares method of fitting, to determine the model parameters directly. In particular, to get more accurate values of the moments $\left.\left\langle\xi^{n}\right\rangle_{2 ; \pi}\right|_{\mu}$, we recalculate these moments with the QCD sum rules in the framework of the background field theory (BFTSR) and adopt a more reasonable and accurate sum rules formula for $\left.\left\langle\xi^{n}\right\rangle_{2 ; \pi}\right|_{\mu}$.

The remaining parts of this paper are organized as follows. In Sec. II A, we recalculate the pionic leadingtwist DA moments using BFTSR. In Sec. II B, we give a brief overview of the LCHO model, put forward the improved new model, and introduce the least squares method to determine the model parameters. Numerical results are given in Sec. III. Section IV is reserved for a summary.

\section{THEORETICAL FRAMEWORK}

\section{A. BFTSR for the moments of $\phi_{2 ; \pi}(x, \mu)$}

To derive the sum rules for the pionic leading-twist DA moments $\left.\left\langle\xi^{n}\right\rangle_{2 ; \pi}\right|_{\mu}$, we adopt the following correlation function:

$$
\begin{aligned}
\Pi_{2 ; \pi}^{(n, 0)}(z, q) & =i \int d^{4} x e^{i q \cdot x}\left\langle 0\left|T\left\{J_{n}(x) J_{0}^{\dagger}(0)\right\}\right| 0\right\rangle \\
& =(z \cdot q)^{n+2} I_{2 ; \pi}^{(n, 0)}\left(q^{2}\right),
\end{aligned}
$$

where $z^{2}=0, n=(0,2,4, \ldots)$ since the odd moments vanish due to the isospin symmetry, and the currents

$$
\begin{gathered}
J_{n}(x)=\bar{d}(x) \not \gamma_{5}(i z \cdot \stackrel{\leftrightarrow}{D})^{n} u(x), \\
J_{0}^{\dagger}(0)=\bar{u}(0) \not \gamma_{5} d(0) .
\end{gathered}
$$

In the physical region, the correlation function (1) can be calculated by inserting a complete set of intermediate hadronic states. Combining the definition

$$
\left\langle 0\left|\bar{d}(0) \not \gamma_{5}(i z \cdot \stackrel{\leftrightarrow}{D})^{n} u(0)\right| \pi(q)\right\rangle=\left.i(z \cdot q)^{n+1} f_{\pi}\left\langle\xi^{n}\right\rangle_{2 ; \pi}\right|_{\mu}
$$

and the quark-hadron duality, the hadron expression of Eq. (1) can be obtained as

$$
\begin{aligned}
\operatorname{Im} I_{2 ; \pi \text { had }}^{(n, 0)}\left(q^{2}\right)= & \left.\pi \delta\left(q^{2}-m_{\pi}^{2}\right) f_{\pi}^{2}\left\langle\xi^{n}\right\rangle_{2 ; \pi}\right|_{\mu} \\
& +\pi \frac{3}{4 \pi^{2}(n+1)(n+3)} \theta\left(q^{2}-s_{\pi}\right),
\end{aligned}
$$

where $m_{\pi}$ is the pion mass, $f_{\pi}$ is the decay constant, and $s_{\pi}$ stands for the continuum threshold. In Eq. (4), the moments $\left.\left\langle\xi^{n}\right\rangle_{2 ; \pi}\right|_{\mu}$ are defined with the pionic leading-twist DA $\phi_{2 ; \pi}(x, \mu)$ as follows:

$$
\left.\left\langle\xi^{n}\right\rangle_{2 ; \pi}\right|_{\mu}=\int_{0}^{1} d x(2 x-1)^{n} \phi_{2 ; \pi}(x, \mu) .
$$

In the deep Euclidean region, we apply the operator product expansion (OPE) for the correlation function (1). The corresponding calculation is performed in the framework of BFTSR. For the basic assumption of BFTSR, the corresponding Feynman rules, and the OPE calculation technology, we refer to Refs. [17,18] for a detailed discussion.

The hadronic expression of the correlation function (1) in the physical region and its OPE in the deep Euclidean region can be matched with the dispersion relation. After applying the Borel transformation for both sides, the sum rules for the moments of the pionic leading-twist DA $\phi_{2 ; \pi}(x, \mu)$ can be obtained as 


$$
\begin{aligned}
\frac{\left.\left.\left\langle\xi^{n}\right\rangle_{2 ; \pi}\right|_{\mu}\left\langle\xi^{0}\right\rangle_{2 ; \pi}\right|_{\mu} f_{\pi}^{2}}{M^{2} e^{m_{\pi}^{2} / M^{2}}}= & \frac{3}{4 \pi^{2}} \frac{1}{(n+1)(n+3)}\left(1-e^{-s_{\pi} / M^{2}}\right)+\frac{\left(m_{d}+m_{u}\right)\langle\bar{q} q\rangle}{\left(M^{2}\right)^{2}}+\frac{\left\langle\alpha_{s} G^{2}\right\rangle}{\left(M^{2}\right)^{2}} \frac{1+n \theta(n-2)}{12 \pi(n+1)} \\
& -\frac{\left(m_{d}+m_{u}\right)\left\langle g_{s} \bar{q} \sigma T G q\right\rangle}{\left(M^{2}\right)^{3}} \frac{8 n+1}{18}+\frac{\left\langle g_{s} \bar{q} q\right\rangle^{2}}{\left(M^{2}\right)^{3}} \frac{4(2 n+1)}{81}-\frac{\left\langle g_{s}^{3} f G^{3}\right\rangle}{\left(M^{2}\right)^{3}} \frac{n \theta(n-2)}{48 \pi^{2}}+\frac{\left\langle g_{s}^{2} \bar{q} q\right\rangle^{2}}{\left(M^{2}\right)^{3}} \frac{2+\kappa^{2}}{486 \pi^{2}} \\
& \times\left\{-2(51 n+25)\left(-\ln \frac{M^{2}}{\mu^{2}}\right)+3(17 n+35)+\theta(n-2)\left[2 n\left(-\ln \frac{M^{2}}{\mu^{2}}\right)+\frac{49 n^{2}+100 n+56}{n}\right.\right. \\
& \left.\left.-25(2 n+1)\left[\psi\left(\frac{n+1}{2}\right)-\psi\left(\frac{n}{2}\right)+\ln 4\right]\right]\right\}
\end{aligned}
$$

where $M$ is the Borel parameter, and for the vacuum condensates we have taken

$$
\begin{aligned}
\langle\bar{q} q\rangle & =\langle\bar{d} d\rangle=\langle\bar{u} u\rangle, \\
\left\langle g_{s} \bar{q} \sigma T G q\right\rangle & =\left\langle g_{s} \bar{d} \sigma T G d\right\rangle=\left\langle g_{s} \bar{u} \sigma T G u\right\rangle, \\
\left\langle g_{s} \bar{q} q\right\rangle^{2} & =\left\langle g_{s} \bar{d} d\right\rangle^{2}=\left\langle g_{s} \bar{u} u\right\rangle^{2}, \\
\left\langle g_{s}^{2} \bar{q} q\right\rangle^{2} & =\left\langle g_{s}^{2} \bar{d} d\right\rangle^{2}=\left\langle g_{s}^{2} \bar{u} u\right\rangle^{2},
\end{aligned}
$$

and with $\langle\bar{s} s\rangle /\langle\bar{q} q\rangle=\kappa$,

$$
g_{s}^{2} \sum\left\langle g_{s} \bar{\psi} \psi\right\rangle^{2}=\left(2+\kappa^{2}\right)\left\langle g_{s}^{2} \bar{q} q\right\rangle^{2}, \quad(\psi=u, d, s) .
$$

In the OPE calculation for the correlation function (1), we have corrected the mistake of a vacuum matrix element, $\left\langle 0\left|G_{\mu \nu}^{A} G_{\rho \sigma ; \lambda \tau}^{B}\right| 0\right\rangle$, used in the previous work [18]. That is,

$$
\begin{aligned}
\left\langle 0\left|G_{\mu \nu}^{A} G_{\rho \sigma ; \lambda \tau}^{B}\right| 0\right\rangle= & \delta^{A B}\left\{( - \frac { 1 } { 1 2 9 6 } \sum \langle g _ { s } \overline { \psi } \psi \rangle ^ { 2 } - \frac { 1 } { 3 8 4 } \langle g _ { s } f G ^ { 3 } \rangle ) \left[2 g_{\lambda \tau}\left(g_{\mu \sigma} g_{\nu \rho}-g_{\mu \rho} g_{\nu \sigma}\right)+g_{\rho \tau}\left(g_{\mu \sigma} g_{\nu \lambda}-g_{\mu \lambda} g_{\nu \sigma}\right)\right.\right. \\
& \left.+g_{\sigma \tau}\left(g_{\mu \lambda} g_{\nu \rho}-g_{\mu \rho} g_{\nu \lambda}\right)\right]+\left(-\frac{1}{1296} \sum\left\langle g_{s} \bar{\psi} \psi\right\rangle^{2}+\frac{1}{384}\left\langle g_{s} f G^{3}\right\rangle\right)\left[g_{\mu \tau}\left(g_{\rho \nu} g_{\sigma \lambda}-g_{\rho \lambda} g_{\nu \sigma}\right)+g_{\nu \tau}\right. \\
& \left.\left.\times\left(g_{\rho \lambda} g_{\sigma \mu}-g_{\rho \mu} g_{\sigma \lambda}\right)\right]\right\} .
\end{aligned}
$$

It needs to be noted that, by taking $n=0$ in Eq. (6) and considering the normalization of the pionic leading-twist DA $\phi_{2 ; \pi}(x, \mu)$, one can obtain the zeroth moment

$$
\left.\left\langle\xi^{0}\right\rangle_{2 ; \pi}\right|_{\mu}=1
$$

Therefore, in many QCD sum rules calculations people usually substitute Eq. (9) as input directly into the sum rules (7), and take Eq. (7) as the sum rules of the moments $\left.\left\langle\xi^{n}\right\rangle_{2 ; \pi}\right|_{\mu}$. This will give rise to an extra deviation to the predicted values of $\left.\left\langle\xi^{n}\right\rangle_{2 ; \pi}\right|_{\mu}$; the reason is that the zeroth moment $\left.\left\langle\xi^{0}\right\rangle_{2 ; \pi}\right|_{\mu}$ on the lhs of Eq. (7) is not strictly that in Eq. (9). By taking $n=0$ in Eq. (7), one can obtain the sum rule of $\left.\left\langle\xi^{0}\right\rangle_{2 ; \pi}\right|_{\mu}$,

$$
\begin{aligned}
\frac{\left.\left\langle\xi^{0}\right\rangle_{2 ; \pi}^{2}\right|_{\mu} f_{\pi}^{2}}{M^{2} e^{m_{\pi}^{2} / M^{2}}}= & \frac{1}{4 \pi^{2}}\left(1+\frac{\alpha_{s}}{\pi}\right)\left(1-e^{-s_{\pi} / M^{2}}\right)+\left(m_{d}\right. \\
& \left.+m_{u}\right) \frac{\langle\bar{q} q\rangle}{\left(M^{2}\right)^{2}}+\frac{\left\langle\alpha_{s} G^{2}\right\rangle}{\left(M^{2}\right)^{2}} \frac{1}{12 \pi}-\frac{1}{18}\left(m_{d}+m_{u}\right) \\
& \times \frac{\left\langle g_{s} \bar{q} \sigma T G q\right\rangle}{\left(M^{2}\right)^{3}}+\frac{4}{81} \frac{\left\langle g_{s} \bar{q} q\right\rangle^{2}}{\left(M^{2}\right)^{3}}+\frac{\left\langle g_{s}^{2} \bar{q} q\right\rangle^{2}}{\left(M^{2}\right)^{3}} \frac{2+\kappa^{2}}{486 \pi^{2}} \\
& \times\left[-50\left(-\ln \frac{M^{2}}{\mu^{2}}\right)+105\right] .
\end{aligned}
$$

Obviously, $\left.\left\langle\xi^{0}\right\rangle_{2 ; \pi}\right|_{\mu}$ on the lhs of the sum rule (7) cannot be normalized in the whole Borel parameter region. The reason is that our calculation is not complete. The highorder corrections and high-dimensional corrections have 
TABLE I. Expressions of the spin-space wave function $\chi_{2 ; \pi}^{\lambda_{1} \lambda_{2}}\left(x, \mathbf{k}_{\perp}\right)$ with different $\lambda_{1} \lambda_{2}$.

\begin{tabular}{lcccc}
\hline \hline$\lambda_{1} \lambda_{2}$ & $\downarrow \downarrow$ & $\uparrow \uparrow$ & $\uparrow \downarrow$ & $\downarrow \uparrow$ \\
\hline$\chi_{2 ; \pi}^{\lambda_{1} \lambda_{2}}\left(x, \mathbf{k}_{\perp}\right)$ & $-\frac{k_{x}+i k_{y}}{\sqrt{2\left(m_{q}^{2}+\mathbf{k}_{\perp}^{2}\right)}}$ & $-\frac{k_{x}-i k_{y}}{\sqrt{2\left(m_{q}^{2}+\mathbf{k}_{\perp}^{2}\right)}}$ & $\frac{m_{q}}{\sqrt{2\left(m_{q}^{2}+\mathbf{k}_{\perp}^{2}\right)}}$ & $-\frac{m_{q}}{\sqrt{2\left(m_{q}^{2}+\mathbf{k}_{\perp}^{2}\right)}}$ \\
\hline \hline
\end{tabular}

not been calculated, and they are also impossible to calculate completely. In fact, the authors of Ref. [8] discovered this more than 30 years ago. They obtained $\left.\left\langle\xi^{0}\right\rangle_{2 ; \pi}\right|_{\mu} \simeq 0.83$, and took $\left.f_{\pi}\left\langle\xi^{0}\right\rangle_{2 ; \pi}\right|_{\mu}$ as the normalization factor to calculate the values of $\left.\left\langle\xi^{2}\right\rangle_{2 ; \pi}\right|_{\mu}$ and $\left.\left\langle\xi^{4}\right\rangle_{2 ; \pi}\right|_{\mu}$. In this paper, we argue that we need to further consider the impact of the sum rule of $\left.\left\langle\xi^{0}\right\rangle_{2 ; \pi}\right|_{\mu}$, Eq. (10), in the full Borel parameter region when using the sum rule (7) to calculate $\left.\left\langle\xi^{n}\right\rangle_{2 ; \pi}\right|_{\mu}$. Therefore, in order to obtain more accurate moments $\left.\left\langle\xi^{n}\right\rangle_{2 ; \pi}\right|_{\mu}$, we suggest the following form:

$$
\left.\left\langle\xi^{n}\right\rangle_{2 ; \pi}\right|_{\mu}=\frac{\left.\left(\left.\left.\left\langle\xi^{n}\right\rangle_{2 ; \pi}\right|_{\mu}\left\langle\xi^{0}\right\rangle_{2 ; \pi}\right|_{\mu}\right)\right|_{\text {From Eq. (7) }}}{\left.\sqrt{\left.\left\langle\xi^{0}\right\rangle_{2 ; \pi}^{2}\right|_{\mu}}\right|_{\text {From Eq. (10) }}}
$$

Meanwhile, another advantage of Eq. (11) is that it can also eliminate some systematic errors caused by the continuum state, the absence of high-dimensional condensates, and the selection and determination of various input parameters.

It should be mentioned that Eq. (10) is usually used to predict the pion decay constant $f_{\pi}$ based on the premise that $\left.\left\langle\xi^{0}\right\rangle_{2 ; \pi}\right|_{\mu} \equiv 1$. After the previous discussion, we think that the sum rule of $\left.\left\langle\xi^{0}\right\rangle_{2 ; \pi}\right|_{\mu}$ varies with the Borel parameter $M^{2}$, especially when $f_{\pi}$ has a definite experimental value. In order to ensure the QCD sum rule's predictive ability for other meson decay constants, we need to assume that $\left.\left\langle\xi^{0}\right\rangle_{2 ; \pi}\right|_{\mu}$ can be normalized in an appropriate Borel window.

\section{B. Improved LCHO model for $\phi_{2 ; \pi}(x, \mu)$}

Based on the BHL description [15], the LCHO model of the pion leading-twist WF was derived in Refs. $[19,20]$ and its form is

$$
\Psi_{2 ; \pi}\left(x, \mathbf{k}_{\perp}\right)=\sum_{\lambda_{1} \lambda_{2}} \chi_{2 ; \pi}^{\lambda_{1} \lambda_{2}}\left(x, \mathbf{k}_{\perp}\right) \Psi_{2 ; \pi}^{R}\left(x, \mathbf{k}_{\perp}\right),
$$

where $\mathbf{k}_{\perp}$ is the pionic transverse momentum, and $\lambda_{1}$ and $\lambda_{2}$ are the helicities of the two constituent quarks. $\chi_{2 ; \pi}^{\lambda_{1} \lambda_{2}}\left(x, \mathbf{k}_{\perp}\right)$ stands for the spin-space WF that comes from the WignerMelosh rotation, whose explicit forms for different $\lambda_{1} \lambda_{2}$ are exhibited in Table I, and which can also been seen in Refs. [21-24].

$$
\Psi_{2 ; \pi}^{R}\left(x, \mathbf{k}_{\perp}\right)=A_{2 ; \pi} \varphi_{2 ; \pi}(x) \exp \left[-\frac{\mathbf{k}_{\perp}^{2}+m_{q}^{2}}{8 \beta_{2 ; \pi}^{2} x \bar{x}}\right],
$$

indicates the spatial WF, where $\bar{x}=1-x, A_{2 ; \pi}$ is the normalization constant, the $\mathbf{k}_{\perp}$-dependent part of the spatial $\mathrm{WF} \Psi_{2 ; \pi}^{R}\left(x, \mathbf{k}_{\perp}\right)$ comes from the approximate bound-state solution in the quark model for the pion [25] and determines the WF's transverse distribution via the harmonious parameter $\beta_{2 ; \pi}$, while the $x$-dependent part $\varphi_{2 ; \pi}(x)$ dominates the WF's longitudinal distribution. In principle, the spatial WF $\Psi_{2 ; \pi}^{R}\left(x, \mathbf{k}_{\perp}\right)$ should include a Jacobi factor. The numerical calculation in Sec. III C will show that the Jacobi factor has little effect on the behavior of the pionic leading-twist DA. In Table I and Eq. (13), $m_{q}$ stands for the mass of the constitute quarks $u$ and $d$ in the pion. In our previous work [26], the experimental data of the pion-photon transition form factor reported by the CELLO, CLEO, BABAR, and BELLE collaborations based on the LCHO model with the longitudinal distribution function $\varphi_{2 ; \pi}^{\mathrm{I}}(x)$ [see Eq. (18)] were fit by adopting the least squares method. We take the constituent quark mass $m_{q}$ and the model parameter $B$ as the fitting parameters, and obtain $m_{q}=(216,246,347,222) \mathrm{MeV}$ for CELLO, CLEO, BABAR, and BELLE data, respectively. The corresponding goodness of fit values are $P_{\chi_{\min }^{2}} / n_{d}=(0.187 / 3,0.986 / 13,0.416 / 15,0.958 / 13)$. We take $m_{q}=200 \mathrm{MeV}$ in this paper. Otherwise, $m_{q}$ is taken to be $250 \mathrm{MeV}$ in the invariant meson mass scheme [27-33] and $330 \mathrm{MeV}$ in the spin-averaged meson mass scheme [34-38]. Therefore, we will discuss the impact of different values of $m_{q}$ on the behavior of our pionic leading-twist DA in detail by taking $m_{q}=200-350 \mathrm{MeV}$.

Using the relationship between the pionic leading-twist DA and WF,

$$
\phi_{2 ; \pi}(x, \mu)=\frac{2 \sqrt{6}}{f_{\pi}} \int_{\left|\mathbf{k}_{\perp}\right|^{2} \leq \mu^{2}} \frac{d^{2} \mathbf{k}_{\perp}}{16 \pi^{3}} \Psi_{2 ; \pi}\left(x, \mathbf{k}_{\perp}\right),
$$

the leading-twist DA for the pion, $\phi_{2 ; \pi}(x, \mu)$, can be obtained. That is, after integrating over the transverse momentum $\mathbf{k}_{\perp}$ in Eq. (14), we have

$$
\begin{aligned}
\phi_{2 ; \pi}(x, \mu)= & \frac{\sqrt{3} A_{2 ; \pi} m_{q} \beta_{2 ; \pi}}{2 \pi^{3 / 2} f_{\pi}} \sqrt{x \bar{x}} \varphi_{2 ; \pi}(x) \\
& \times\left\{\operatorname{Erf}\left[\sqrt{\frac{m_{q}^{2}+\mu^{2}}{8 \beta_{2 ; \pi}^{2} x \bar{x}}}\right]-\operatorname{Erf}\left[\sqrt{\frac{m_{q}^{2}}{8 \beta_{2 ; \pi}^{2} x \bar{x}}}\right]\right\},
\end{aligned}
$$


where $\operatorname{Erf}(x)=2 \int_{0}^{x} e^{-t^{2}} d x / \sqrt{\pi}$ is the error function. The error function part in Eq. (15) comes from the $\mathbf{k}_{\perp^{-}}$ dependent part of the WF $\Psi_{2 ; \pi}\left(x, \mathbf{k}_{\perp}\right)$ and gives a good end-point behavior for $\phi_{2 ; \pi}(x, \mu)$, and $\varphi_{2 ; \pi}(x)$ dominates the broadness of $\phi_{2 ; \pi}(x, \mu)$. Obviously, the specific form of $\phi_{2 ; \pi}(x, \mu)$ is determined by the parameters $A_{2 ; \pi}$ and $\beta_{2 ; \pi}$ and the function $\varphi_{2 ; \pi}(x)$. There are two important constraints [15] which can be used to constrain the parameters $A_{2 ; \pi}$ and $\beta_{2 ; \pi}$ :

(1) The WF normalization condition provided from the process $\pi \rightarrow \mu \nu$,

$$
\int_{0}^{1} d x \int \frac{d^{2} \mathbf{k}_{\perp}}{16 \pi^{3}} \Psi\left(x, \mathbf{k}_{\perp}\right)=\frac{f_{\pi}}{2 \sqrt{6}} .
$$

(2) The sum rule derived from the $\pi^{0} \rightarrow \gamma \gamma$ decay amplitude,

$$
\int_{0}^{1} d x \Psi\left(x, \mathbf{k}_{\perp}=\mathbf{0}\right)=\frac{\sqrt{6}}{f_{\pi}} .
$$

Then, the pionic leading-twist DA $\phi_{2 ; \pi}(x, \mu)$ only depends on the mathematical form of $\varphi_{2 ; \pi}(x)$. By solving the renormalization group equation of the pionic leadingtwist DA, $\phi_{2 ; \pi}(x, \mu)$ can be written as the expansion form of the Gegenbauer series [2,3]. Based on this, in our previous paper $\varphi_{2 ; \pi}(x)$ was taken to be the linear superposition of the first several Gegenbauer polynomials. For example, in Refs. [26,39-41] we took

$$
\varphi_{2 ; \pi}^{\mathrm{I}}(x)=1+B \times C_{2}^{3 / 2}(2 x-1),
$$

and

$$
\begin{aligned}
\varphi_{2 ; \pi}^{\mathrm{II}}(x)= & 1+B_{2} \times C_{2}^{3 / 2}(2 x-1) \\
& +B_{4} \times C_{4}^{3 / 2}(2 x-1)
\end{aligned}
$$

was adopted in Ref. [18]. For the former, when the value of the parameter $B$ changes from 0.0 to 0.6 , the pionic leadingtwist DA model, i.e., Eq. (15) can mimic the DA behavior from asymptotic-like to CZ-like. For the latter, we further consider the correction of a fourth-order Gegenbauer polynomial. The mathematical form of $\varphi_{2 ; \pi}(x)$ can usually be determined in two ways. The first one is to extract $\varphi_{2 ; \pi}(x)$ from the experimental data of the exclusive processes involving a pion [26,39-41], such as the semileptonic decays $B \rightarrow \pi \ell \nu_{\ell}$ and $D \rightarrow \pi \ell \nu_{\ell}$, the pion-photon transition form factor $F_{\pi \gamma}\left(Q^{2}\right)$, and the exclusive process $B^{0} \rightarrow \pi^{0} \pi^{0}$. The second one is to determine $\varphi_{2 ; \pi}(x)$ from the moments $\left.\left\langle\xi^{n}\right\rangle_{2 ; \pi}\right|_{\mu}$ or the Gegenbauer moments $a_{n}^{2 ; \pi}(\mu)$ of $\phi_{2 ; \pi}(x, \mu)$. In Ref. [18], we adopted the second method to determine the mathematical form of $\varphi_{2 ; \pi}(x)$ and further the behavior of $\phi_{2 ; \pi}(x, \mu)$.
In this paper, we will still make use of the second method mentioned above to determine the behavior of $\phi_{2 ; \pi}(x, \mu)$, but we will improve it. The accuracy of the behavior of $\phi_{2 ; \pi}(x, \mu)$ obtained using this method is restricted by two aspects: the rationality of the constructed mathematical form of $\varphi_{2 ; \pi}(x)$ and the accuracy of moments. In order to obtain a better mathematical form of $\varphi_{2 ; \pi}(x)$, a natural idea is to add higherorder Gegenbauer polynomial corrections in $\varphi_{2 ; \pi}^{\mathrm{II}}(x)$, as we have done for the $D, \eta_{c}, B_{c}, \eta_{b}$ twist-2 and -3 DAs in Refs. [42-45]. However, such an improvement obviously destroys the beauty and conciseness of the model. Otherwise, we find that the parameters $B_{2}$ and $B_{4}$ are close to the Gegenbauer moments $a_{2}^{2 ; \pi}(\mu)$ and $a_{4}^{2 ; \pi}(\mu)$, respectively. From the relationship between $\left.\left\langle\xi^{n}\right\rangle_{2 ; \pi}\right|_{\mu}$ and $a_{n}^{2 ; \pi}(\mu)$, it can be seen that the reliability of $a_{n}^{2 ; \pi}(\mu)$ calculated using QCD sum rules decreases sharply with the increase of order $n$. In view of this, in this paper we will improve the mathematical form of $\varphi_{2 ; \pi}(x)$ in another way, as well as propose a new method of determining model parameters.

We notice that although it is difficult to improve the pionic leading-twist DA by introducing higher Gegenbauer polynomial corrections, our goal is still to make it more reasonable and accurate by adjusting the behavior of $\phi_{2 ; \pi}(x, \mu)$. We find that the factor $\sqrt{x \bar{x}}$ in Eq. (15) can regulate the DA's behavior to some extent. Inspired by this, we introduce a factor $[x \bar{x}]^{\alpha_{2 ; \pi}}$ into the WF's longitudinal distribution function $\varphi_{2 ; \pi}(x)$, i.e.,

$$
\varphi_{2 ; \pi}^{\mathrm{III}}(x)=[x \bar{x}]^{\alpha_{2 ; \pi}} .
$$

In order to further apply our LCHO model to other meson DAs, by combining the form of $\varphi_{2 ; \pi}^{\mathrm{I}}(x)$ and $\varphi_{2 ; \pi}^{\mathrm{III}}(x)$, we propose a more complex form,

$$
\varphi_{2 ; \pi}^{\mathrm{IV}}(x)=[x \bar{x}]^{\alpha_{2 ; \pi}[1}\left[1+\hat{a}_{2}^{2 ; \pi} C_{2}^{3 / 2}(2 x-1)\right],
$$

where the parameters $\alpha_{2 ; \pi}$ and $\hat{a}_{2}^{2 ; \pi}$ will be determined by fitting the moments $\left.\left\langle\xi^{n}\right\rangle_{2 ; \pi}\right|_{\mu}$ directly through the method of least squares, and the values of the moments $\left.\left\langle\xi^{n}\right\rangle_{2 ; \pi}\right|_{\mu}$ come from Eq. (11) calculated under BFTSR in Sec. II A. In order to distinguish our LCHO model with $\varphi_{2 ; \pi}^{\mathrm{III}}(x)$ and $\varphi_{2 ; \pi}^{\mathrm{IV}}(x)$, and facilitate the discussion later, we will denote the former as LCHO model-III and the latter as LCHO model-IV.

Considering a set of $N$ independent measurements $y_{i}$ with known variance $\sigma_{i}$ and mean $\mu\left(x_{i} ; \boldsymbol{\theta}\right)$ at known points $x_{i}$, the objective of the least squares method is to obtain the best value of the fitting parameters $\boldsymbol{\theta}$ by minimizing the likelihood function [46]

$$
\chi^{2}(\boldsymbol{\theta})=\sum_{i=1}^{N} \frac{\left(y_{i}-\mu\left(x_{i}, \boldsymbol{\theta}\right)\right)^{2}}{\sigma_{i}^{2}} .
$$


As for the present case, the function $\mu\left(x_{i} ; \boldsymbol{\theta}\right)$ indicates the pionic leading-twist DA moments $\left.\left\langle\xi^{n}\right\rangle_{2 ; \pi}\right|_{\mu}$ defined by combining Eqs. (6), (15), and (20) and $\boldsymbol{\theta}=\left(\alpha_{2 ; \pi}, \hat{a}_{2}^{2 ; \pi}\right)$; the theoretical values of $\left.\left\langle\xi^{n}\right\rangle_{2 ; \pi}\right|_{\mu}$ calculated using QCD sum rules in next section are assumed to be the value of $y_{i}$ and its variance $\sigma_{i}$. The probability density function of $\chi^{2}$ can be obtained,

$$
f\left(y ; n_{d}\right)=\frac{1}{\Gamma\left(\frac{n_{d}}{2}\right) 2^{n_{d} / 2}} y^{\frac{n_{d}}{2}-1} e^{-\frac{y}{2}}
$$

where $n_{d}$ is the number of degrees of freedom. Then, one can further calculate the following probability:

$$
P_{\chi^{2}}=\int_{\chi^{2}}^{\infty} f\left(y ; n_{d}\right) d y
$$

The magnitude of the probability $P_{\chi^{2}}\left(P_{\chi^{2}} \in[0,1]\right)$ can be used to judge the goodness of fit: when its value is closer to 1 , a better fit is assumed to be achieved.

\section{NUMERICAL ANALYSIS}

\section{A. Basic input parameters}

To do the numerical calculation, we adopt the latest data from the Particle Data Group [46]: $m_{\pi}=139.57039 \pm$ $0.00017 \mathrm{MeV}$ and $f_{\pi}=130.2 \pm 1.2 \mathrm{MeV}$. The currentquark masses for the $u, d$ quarks are adopted as $m_{u}=2.16_{-0.26}^{+0.49} \mathrm{MeV}$ and $m_{d}=4.67_{-0.17}^{+0.48} \mathrm{MeV}$ at the scale $\mu=2 \mathrm{GeV}$. Based on these latest values, we can update the vacuum condensates.

(1) For the double-quark condensate, we adopt the GellMann-Oakes-Renner relation:

$$
\begin{aligned}
& m_{u}\langle\bar{u} u\rangle+m_{d}\langle\bar{d} d\rangle \simeq-\frac{f_{\pi}^{2} m_{\pi}^{2}}{2} \\
& \quad=-(1.651 \pm 0.003) \times 10^{-4} \mathrm{GeV}^{4}
\end{aligned}
$$

Combined with the $u, d$ quark masses, we have

$$
\begin{aligned}
\langle\bar{q} q\rangle & =\left(-2.417_{-0.114}^{+0.227}\right) \times 10^{-2} \mathrm{GeV}^{3} \\
& =\left(-289.14_{-4.47}^{+9.34}\right)^{3} \mathrm{MeV}^{3}
\end{aligned}
$$

at the scale $\mu=2 \mathrm{GeV}$.

(2) By combining Eqs. (25) and (26) and the relation $\left\langle g_{s} \bar{q} \sigma T G q\right\rangle=m_{0}^{2}\langle\bar{q} q\rangle$ with $m_{0}^{2}=0.80 \pm 0.02 \mathrm{GeV}^{2}$ [47], the quark-gluon mixed condensate is

$$
\begin{gathered}
m_{u}\left\langle g_{s} \bar{u} \sigma T G u\right\rangle+m_{d}\left\langle g_{s} \bar{d} \sigma T G d\right\rangle \\
=-(1.321 \pm 0.033) \times 10^{-4} \mathrm{GeV}^{6}, \\
\left\langle g_{s} \bar{q} \sigma T G q\right\rangle=\left(-1.934_{-0.103}^{+0.188}\right) \times 10^{-2} \mathrm{GeV}^{5} .
\end{gathered}
$$

(3) By adopting the data in Ref. [47],

$$
\rho \alpha_{s}\langle\bar{q} q\rangle^{2}=(5.8 \pm 1.8) \times 10^{-4} \mathrm{GeV}^{6},
$$

with $\rho \simeq 3-4$, combined with the value of the double-quark condensate in Eq. (26), the four-quark condensates can be obtained as

$$
\left\langle g_{s} \bar{q} q\right\rangle^{2}=\left(2.082_{-0.697}^{+0.734}\right) \times 10^{-3} \mathrm{GeV}^{6}
$$

and

$$
\left\langle g_{s}^{2} \bar{q} q\right\rangle^{2}=\left(7.420_{-2.483}^{+2.614}\right) \times 10^{-3} \mathrm{GeV}^{6} .
$$

(4) From Ref. [48], we have

$$
\left\langle\alpha_{s} G^{2}\right\rangle=0.038 \pm 0.011 \mathrm{GeV}^{4}
$$

and

$$
\left\langle g_{s}^{3} f G^{3}\right\rangle \simeq 0.045 \mathrm{GeV}^{6} .
$$

(5) For the ratio $\kappa=\langle\bar{s} s\rangle /\langle\bar{q} q\rangle$, Ref. [49] gives

$$
\kappa=0.74 \pm 0.03 \text {. }
$$

\section{B. Renormalization group equation for the input parameters and moments $\left.\left\langle\xi^{n}\right\rangle_{2 ; \pi}\right|_{\mu}$}

In the numerical calculation of the moments' BFTSR (11), we take the scale $\mu=M$ as usual. From $\alpha_{s}\left(M_{z}\right)=$ $0.1179 \pm 0.0010$ with $M_{Z}=91.1876 \pm 0.0021 \mathrm{GeV}$, and combined with $\bar{m}_{c}\left(\bar{m}_{c}\right)=1.27 \pm 0.02 \mathrm{GeV}$ and $\bar{m}_{b}\left(\bar{m}_{b}\right)=$ $4.18_{-0.02}^{+0.03} \mathrm{GeV}$ [46], under the three-loop approximate solution we predict $\Lambda_{\mathrm{QCD}}^{\left(n_{f}\right)} \simeq 324,286,207 \mathrm{MeV}$ for the number of quark flavors $n_{f}=3,4,5$, respectively.

The renormalization group equations (RGEs) of the quark mass and vacuum condensates are given as [50-52]

$$
\begin{aligned}
\left.m_{q}\right|_{\mu} & =\left.m_{q}\right|_{\mu_{0}}\left[\frac{\alpha_{s}\left(\mu_{0}\right)}{\alpha_{s}(\mu)}\right]^{-4 / \beta_{0}}, \\
\left.\langle\bar{q} q\rangle\right|_{\mu} & =\left.\langle\bar{q} q\rangle\right|_{\mu_{0}}\left[\frac{\alpha_{s}\left(\mu_{0}\right)}{\alpha_{s}(\mu)}\right]^{4 / \beta_{0}}, \\
\left.\left\langle g_{s} \bar{q} \sigma T G q\right\rangle\right|_{\mu} & =\left.\left\langle g_{s} \bar{q} \sigma T G q\right\rangle\right|_{\mu_{0}}\left[\frac{\alpha_{s}\left(\mu_{0}\right)}{\alpha_{s}(\mu)}\right]^{-2 /\left(3 \beta_{0}\right)}, \\
\left.\left\langle\alpha_{s} G^{2}\right\rangle\right|_{\mu} & =\left.\left\langle\alpha_{s} G^{2}\right\rangle\right|_{\mu_{0}}, \\
\left.\left\langle g_{s}^{3} f G^{3}\right\rangle\right|_{\mu} & =\left.\left\langle g_{s}^{3} f G^{3}\right\rangle\right|_{\mu_{0}},
\end{aligned}
$$

with $\beta_{0}=\left(33-2 n_{f}\right) / 3$. Obviously, the double-gluon condensate and the triple-gluon condensate are energyscale independent. From Eq. (8), one can find that $\left\langle g_{s}^{2} \bar{q} q\right\rangle^{2}$ and $\left\langle g_{s}^{3} f G^{3}\right\rangle$ have the same RGE. In other words, $\left\langle g_{s}^{2} \bar{q} q\right\rangle^{2}$ is also energy-scale independent, e.g., 


$$
\left.\left\langle g_{s}^{2} \bar{q} q\right\rangle^{2}\right|_{\mu}=\left.\left\langle g_{s}^{2} \bar{q} q\right\rangle^{2}\right|_{\mu_{0}} .
$$

Combined with the RGE of the double-quark condensate and Eq. (36), one can find that $\left\langle g_{s} \bar{q} q\right\rangle^{2}$ and $\langle\bar{q} q\rangle$ have the same energy-scale evolution equation, e.g.,

$$
\left.\left\langle g_{s} \bar{q} q\right\rangle^{2}\right|_{\mu}=\left.\left\langle g_{s} \bar{q} q\right\rangle^{2}\right|_{\mu_{0}}\left[\frac{\alpha_{s}\left(\mu_{0}\right)}{\alpha_{s}(\mu)}\right]^{4 / \beta_{0}} .
$$

It should be noted that, according to the basic assumption of BFTSR, $g_{s}$ in all of the above vacuum condensates is the "coupling constant" between the background fields, which is different from the one in $\mathrm{pQCD}$, and should be absorbed into vacuum condensates as part of these nonperturbative parameters.

The RGE of the Gegenbauer moments of the pion leading-twist distribution amplitude is

$$
a_{n}^{2 ; \pi}(\mu)=a_{n}^{2 ; \pi}\left(\mu_{0}\right) E_{n}\left(\mu, \mu_{0}\right)
$$

with

$$
E_{n}\left(\mu, \mu_{0}\right)=\left[\frac{\alpha_{s}(\mu)}{\alpha_{s}\left(\mu_{0}\right)}\right]^{\gamma_{n}^{(0)} /\left(2 \beta_{0}\right)}
$$

The leading-order (LO) anomalous dimension is

$$
\gamma_{n}^{(0)}=8 C_{F}\left[\psi(n+2)+\gamma_{E}-\frac{3}{4}-\frac{1}{2(n+1)(n+2)}\right],
$$

with $C_{F}=4 / 3$. Based on Eq. (38), the RGE of the moments $\left.\left\langle\xi^{n}\right\rangle_{2 ; \pi}\right|_{\mu}$ can be obtained.

With the BFTSR of the moments of the pionic leadingtwist distribution amplitude $\phi_{2 ; \pi}(x, \mu)$ shown in Eqs. (7), (10), and (11), the values of $\left.\left\langle\xi^{n}\right\rangle_{2 ; \pi}\right|_{\mu}$ can be calculated. By requiring that there is a reasonable Borel window to normalize $\left.\left\langle\xi^{0}\right\rangle_{2 ; \pi}\right|_{\mu}$ with Eq. (10), one can get the continuum threshold parameter as $s_{\pi} \simeq 1.05 \mathrm{GeV}^{2}$. In addition to the traditional method to determine the contribution of the continuum state, the continuum method can limit or overcome model dependence and cleanly connect the data with QCD itself [53]. To obtain the allowable Borel window for the sum rules of $\left.\left\langle\xi^{n}\right\rangle_{2 ; \pi}\right|_{\mu}$, we require that the continuum state's contribution and the dimension-six condensate's contribution be as small as possible, and the values for $\left.\left\langle\xi^{n}\right\rangle_{2 ; \pi}\right|_{\mu}$ be stable in the Borel window. Based on the criteria, the dimension-six contribution for $\left.\left\langle\xi^{n}\right\rangle_{2 ; \pi}\right|_{\mu}$ are limited to less than $5 \%$ for all the $n$ th-order. And the continuum contribution for $\left.\left\langle\xi^{n}\right\rangle_{2 ; \pi}\right|_{\mu}$ are required no more than $(30,35,40,40,40) \%$ for $n=(2,4,6,8,10)$, respectively.

To have a deeper insight into the continuum state and dimension-six contributions to the pionic leading-twist DA moments $\left.\left\langle\xi^{n}\right\rangle_{2 ; \pi}\right|_{\mu}$ versus the Borel parameter $M^{2}$ within the BFTSR approach, we present the curves in Fig. 1.
The shaded bands indicate the Borel window for $\left.\left\langle\xi^{n}\right\rangle_{2 ; \pi}\right|_{\mu}$ for $n=(2,4,6,8,10)$, respectively. The figure indicates the following.

(1) The dimension-six contributions are constraint in the region $<5 \%$ guaranteed good convergence for the BFTSR results. And the continuum contributions no more than $40 \%$ have agreement with the traditional sum rule strictly.

(2) Borel parameters associated with the region of the Borel window become larger with the increase of the index $n$.

To study the influence of the Borel parameters on the pionic DA moments in the Borel window, we list the results $\left.\left\langle\xi^{n}\right\rangle_{2 ; \pi}\right|_{\mu}$ changed with Borel windows in Table II, in which the $\left.\left\langle\xi^{n}\right\rangle_{2 ; \pi}\right|_{\mu}$ changed less than $10 \%$ with the Borel windows, i.e., $4.1 \%, 6.1 \%, 8.6 \%, 8.8 \%, 7.1 \%$ for $n=(2,4,6,8,10)$, respectively. Thus, the sum rules of $\left.\left\langle\xi^{n}\right\rangle_{2 ; \pi}\right|_{\mu}$ are stable in the Borel windows. Furthermore, the five curves for the pionic leading-twist DA moments, i.e., $\left.\left\langle\xi^{n}\right\rangle_{2 ; \pi}\right|_{\mu}$ for $n=(2,4,6,8,10)$ versus the Borel parameter $M^{2}$ are shown in Fig. 2. The figure indicates the following.

(1) The curves for $\left.\left\langle\xi^{n}\right\rangle_{2 ; \pi}\right|_{\mu}$ change sharply in the small Borel area, especially for the $M^{2} \rightsquigarrow 1 \mathrm{GeV}^{2}$.

(2) The values of $\left.\left\langle\xi^{n}\right\rangle_{2 ; \pi}\right|_{\mu}$ become small with the increase of order $n$.

(3) The stable Borel parameter $M^{2}$ for $\left.\left\langle\xi^{n}\right\rangle_{2 ; \pi}\right|_{\mu}$ becomes larger with the increase of $n$.

After taking all uncertainty sources into consideration and adopting the RGE of moments mentioned in the above subsection, the first five nonvanishing values of $\left.\left\langle\xi^{n}\right\rangle_{2 ; \pi}\right|_{\mu}$, i.e., $n=(2,4,6,8,10)$ within uncertainties coming from every input parameter are shown in Table III. In which, the scale are taken both the initial scale $\mu_{0}$ and typical scale $\mu=2 \mathrm{GeV}$. As a deeper comparison, we also list those moments obtained by the light-front (LF) holographic with $B=0$ and $B \gg 1[54,55]$, platykurtic [56], LF quark model [57], QCD sum rules [5,58], renormalon model [59], instanton vacuum $[60,61]$, nonlocal condensate (NLC) sum rules [62], Dyson-Schwinger [RL,DB] [14], lattice [63-68]. At the same time, we also provide the inverse moment $\left.\left\langle x^{-1}\right\rangle\right|_{\mu}=\int_{0}^{1} d x x^{-1} \phi_{2 ; \pi}(x, \mu)$ in Table III. In addition, in order to show the advantages of the new sum rules formula (11), the values of $\left.\left\langle\xi^{n}\right\rangle_{2 ; \pi}\right|_{\mu}$ obtained using the formula combined with Eqs. (7) and (9) commonly used in literature are also listed in this table. From the table, we can make the following conclusions.

(1) Up to tenth-order accuracy, we provide a complete series result for $\left.\left\langle\xi^{n}\right\rangle_{2 ; \pi}\right|_{\mu}$ within uncertainties.

(2) For the $n=(2,4)$ cases, our results have good agreement with the DS model and lattice results.

(3) The inverse moment at $\mu=2 \mathrm{GeV}$ of our prediction is close to the platykurtic and NLC sum rules results.

(4) Comparing the values in the first and last rows, one can find that the differences between corresponding 

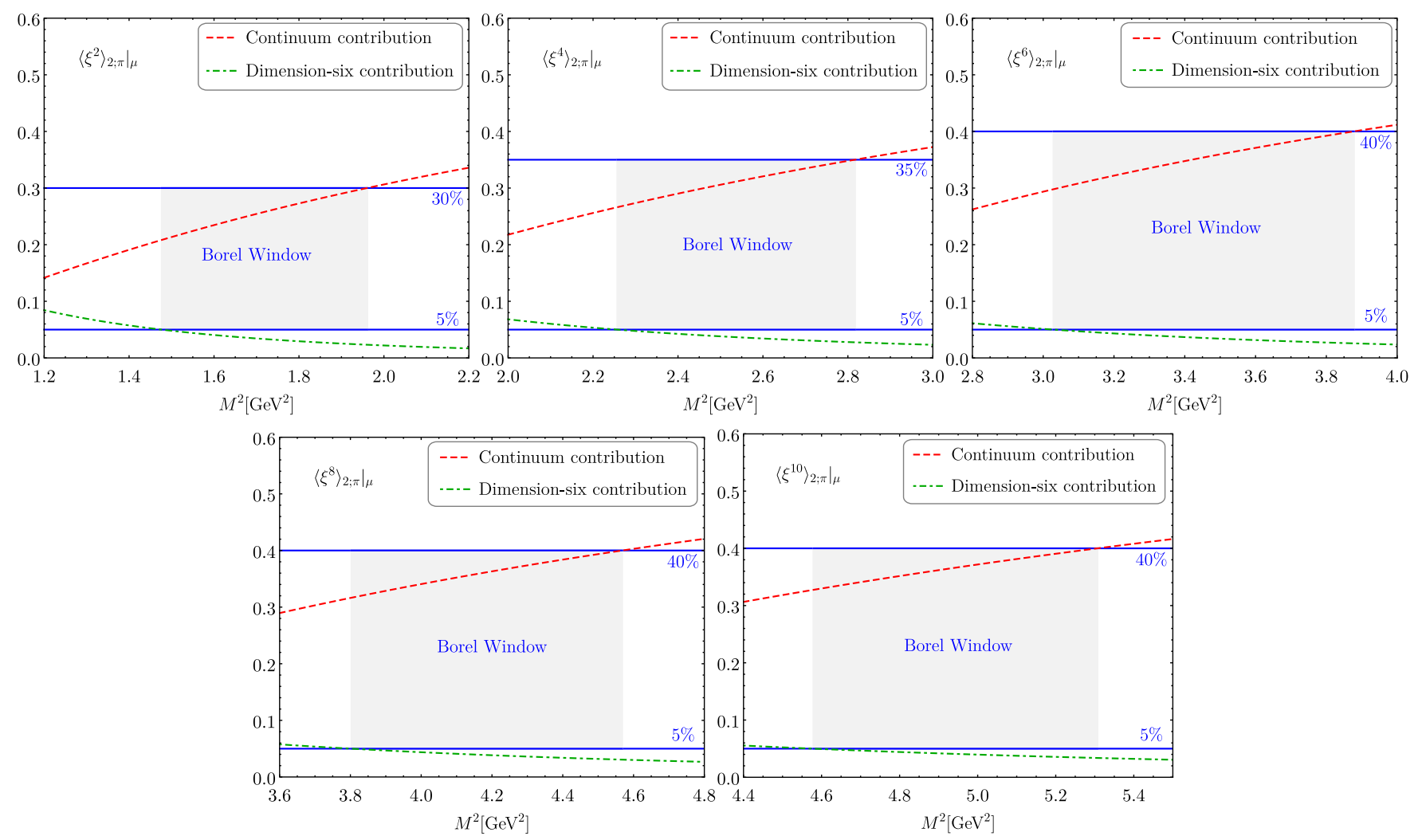

FIG. 1. Continuum state and dimension-six condensate contribute to the pionic leading-twist DA moments $\left.\left\langle\xi^{n}\right\rangle_{2 ; \pi}\right|_{\mu}$ versus the Borel parameter $M^{2}$ within the BFTSR approach. The shaded bands indicate the Borel window for $\left.\left\langle\xi^{n}\right\rangle_{2 ; \pi}\right|_{\mu}$ for $n=(2,4,6,8,10)$, respectively.

moments are about $12 \%, 30 \%, 47 \%, 53 \%$, and $64 \%$ for $n=2,4,6,8,10$, respectively. These ratios can be regarded as the improvement of accuracy due to the adoption of the new sum rules formula (11). At the same time, one can find that these differences increase with the increase of the order $n$. The reason is that the Borel window moves to the right with the increase of order $n$ (see Table II), and the deviation of the sum rule of the zeroth moment [Eq. (10)] from normalization increases with the increase of Borel parameter. The errors in the first row are significantly less than those in the last row. The reason is that the sum rules (11) can eliminate some systematic errors caused by the selection and determination of various input parameters. To calculate $\left.\left\langle\xi^{n}\right\rangle_{2 ; \pi}\right|_{\mu}$ by

TABLE II. Determined Borel windows and corresponding pionic leading-twist DA moments $\left.\left\langle\xi^{n}\right\rangle_{2 ; \pi}\right|_{\mu}$ with $n=(2,4,6,8,10)$. All input parameters are set to their central values.

\begin{tabular}{ccc}
\hline \hline$n$ & $M^{2}$ & $\left.\left\langle\xi^{n}\right\rangle_{2 ; \pi}\right|_{\mu}$ \\
\hline 2 & {$[1.477,1.961]$} & {$[0.268,0.257]$} \\
4 & {$[2.257,2.817]$} & {$[0.132,0.124]$} \\
6 & {$[3.029,3.878]$} & {$[0.081,0.074]$} \\
8 & {$[3.803,4.568]$} & {$[0.057,0.052]$} \\
10 & {$[4.579,5.307]$} & {$[0.042,0.039]$} \\
\hline \hline
\end{tabular}

combining Eqs. (7) and (9), we have required that the continuum state contributions are less than $45 \%$, $50 \%, 50 \%, 55 \%, 55 \%$, and the dimension-six contributions are not more than $10 \%, 15 \%, 15 \%, 15 \%$, $15 \%$, for the order $n=2,4,6,8,10$, respectively. Those criteria are obviously much weaker than that adopted for the sum rules (11) mentioned above,

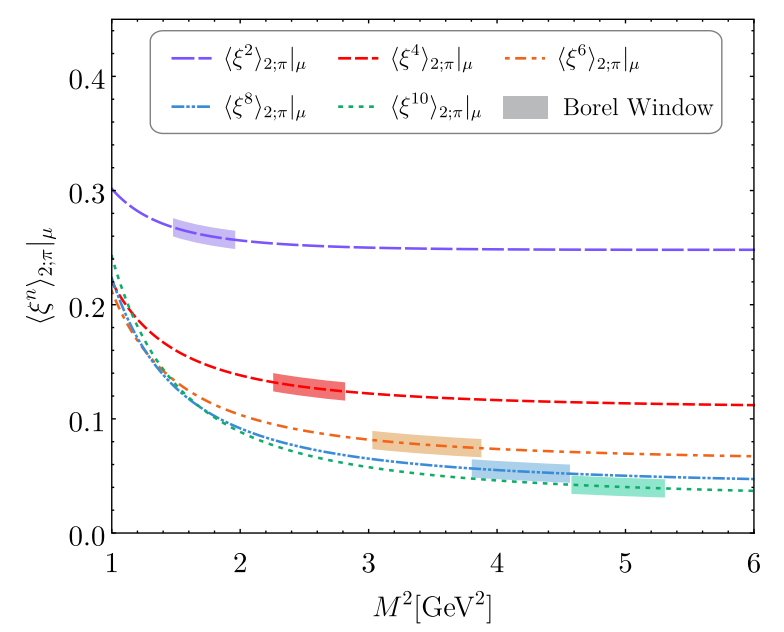

FIG. 2. Pionic leading-twist DA moments $\left.\left\langle\xi^{n}\right\rangle_{2 ; \pi}\right|_{\mu}$ with $n=$ $(2,4,6,8,10)$ versus the Borel parameter $M^{2}$, where all input parameters are set to their central values. The shaded bands indicate the Borel windows for $n=(2,4,6,8,10)$, respectively. 
TABLE III. Our predictions for the first five nonvanishing moments and inverse moment of the pion DA, compared to other theoretical predictions. The values obtained using the formula combining Eqs. (7) and (9) are also shown.

\begin{tabular}{|c|c|c|c|c|c|c|c|}
\hline & $\mu[\mathrm{GeV}]$ & $\left.\left\langle\xi^{2}\right\rangle_{2 ; \pi}\right|_{\mu}$ & $\left.\left\langle\xi^{4}\right\rangle_{2 ; \pi}\right|_{\mu}$ & $\left.\left\langle\xi^{6}\right\rangle_{2 ; \pi}\right|_{\mu}$ & $\left.\left\langle\xi^{8}\right\rangle_{2 ; \pi}\right|_{\mu}$ & $\left.\left\langle\xi^{10}\right\rangle_{2 ; \pi}\right|_{\mu}$ & $\left.\left\langle x^{-1}\right\rangle\right|_{\mu}$ \\
\hline BFTSR (this work) & 1 & $0.271(13)$ & $0.138(10)$ & $0.087(6)$ & $0.064(7)$ & $0.050(6)$ & 3.95 \\
\hline BFTSR (this work) & 2 & $0.254(10)$ & $0.125(7)$ & $0.077(6)$ & $0.054(5)$ & $0.041(4)$ & 3.33 \\
\hline Asymptotic & $\infty$ & 0.200 & 0.086 & 0.048 & 0.030 & 0.021 & 3.00 \\
\hline LF holographic $(B=0)$ [54] & 1,2 & $0.180,0.185$ & $0.067,0.071$ & $\ldots$ & $\ldots$ & $\ldots$ & $2.81,2.85$ \\
\hline LF holographic $(B \gg 1)[54]$ & 1,2 & $0.200,0.200$ & $0.085,0.085$ & $\ldots$ & $\ldots$ & $\ldots$ & $2.93,2.95$ \\
\hline LF holographic [55] & $\sim 1$ & 0.237 & 0.114 & $\ldots$ & $\ldots$ & $\ldots$ & 4.0 \\
\hline Platykurtic [56] & 2 & $0.220_{-0.006}^{+0.009}$ & $0.098_{-0.005}^{+0.008}$ & $\cdots$ & $\ldots$ & .. & $3.13_{-0.10}^{+0.14}$ \\
\hline LF quark model [57] & $\sim 1$ & $0.24(22)$ & $0.11(9)$ & $\ldots$ & $\ldots$ & $\ldots$ & $\ldots$ \\
\hline Sum rules [58] & 1 & 0.24 & 0.11 & $\ldots$ & $\ldots$ & $\ldots$ & $\ldots$ \\
\hline Renormalon model [59] & 1 & 0.28 & 0.13 & $\ldots$ & $\ldots$ & $\ldots$ & $\ldots$ \\
\hline Instanton vacuum $[60,61]$ & 1 & $0.22,0.21$ & $0.10,0.09$ & $\ldots$ & $\ldots$ & $\ldots$ & $\ldots$ \\
\hline NLC sum rules [62] & 2 & $0.248_{-0.015}^{+0.016}$ & $0.108_{-0.03}^{+0.05}$ & $\cdots$ & $\cdots$ & $\cdots$ & $3.16(9)$ \\
\hline Sum rules [5] & 2 & 0.343 & 0.181 & $\ldots$ & $\ldots$ & $\ldots$ & 4.25 \\
\hline Dyson-Schwinger [RL,DB] [14] & 2 & $0.280,0.251$ & $0.151,0.128$ & $\ldots$ & $\ldots$ & $\ldots$ & $5.5,4.6$ \\
\hline Lattice [63] & 2 & $0.28(1)(2)$ & $\ldots$ & $\ldots$ & $\ldots$ & $\ldots$ & $\ldots$ \\
\hline Lattice [64] & 2 & $0.2361(41)(39)$ & $\ldots$ & $\ldots$ & $\ldots$ & $\ldots$ & $\ldots$ \\
\hline Lattice [65] & 2 & $0.27(4)$ & $\ldots$ & $\ldots$ & $\ldots$ & $\ldots$ & $\ldots$ \\
\hline Lattice [66] & 2 & $0.2077(43)$ & $\cdots$ & $\ldots$ & $\ldots$ & $\ldots$ & $\ldots$ \\
\hline Lattice [67] & 2 & $0.234(6)(6)$ & $\ldots$ & $\ldots$ & $\ldots$ & $\ldots$ & $\ldots$ \\
\hline Lattice [68] & 2 & $0.244(30)$ & $\ldots$ & $\cdots$ & $\ldots$ & $\ldots$ & $\cdots$ \\
\hline Eq. (7) + Eq. (9) & 1 & $0.303(19)$ & $0.179(21)$ & $0.128(16)$ & $0.098(14)$ & $0.082(20)$ & $\ldots$ \\
\hline
\end{tabular}

which are obviously much larger. This means that the sum rules (11) eliminate some systematic errors caused by the continuum state and the absence of high-dimensional condensates.

Moreover, considering the low reliability of high-order Gegenbauer moments, we only give the values of the second and forth Gegenbauer moments in this paper, which are shown in Table IV. As a comparison, the values obtained using QCD sum rules [69,70], lattice [63,64,67], light-cone sum rules (LCSR) fitting [71-74], and DysonSchwinger [RL,DB] [14] are also shown. Our predictions agree with the predictions from QCD sum rules, LCSR fitting, and the Dyson-Schwinger equations within errors.

\section{Model parameters of the pionic leading-twist DA and applications}

Combining the normalization condition (16) and the sum rule (17) derived from the $\pi^{0} \rightarrow \gamma \gamma$ decay amplitude, and making use of the least squares method mentioned in Sec. II to fit the values of the moments $\left.\left\langle\xi^{n}\right\rangle_{2 ; \pi}\right|_{\mu}$ shown in Table III, the parameters of our LCHO model-III can be obtained:

TABLE IV. Comparison of the second and fourth Gegenbauer moments of the pion leading-twist DA with different methods.

\begin{tabular}{lccc}
\hline \hline Method & $\mu(\mathrm{GeV})$ & $a_{2}^{2 ; \pi}$ & $a_{4}^{2 ; \pi}$ \\
\hline BFTSR (this work) & 1 & $0.206 \pm 0.038$ & $0.047 \pm 0.011$ \\
BFTSR (this work) & 2 & $0.157 \pm 0.029$ & $0.032 \pm 0.007$ \\
Lattice [63] & 2 & $0.233 \pm 0.065$ & \\
Lattice [64] & 2 & $0.136 \pm 0.021$ & \\
Lattice [67] & 1 & $0.135 \pm 0.032$ & \\
Lattice [67] & 2 & $0.101 \pm 0.023$ & $-0.143_{-0.087}^{+0.094}$ \\
Sum rules [69,70] & 1 & $0.203_{-0.057}^{+0.069}$ & $-0.096_{-0.058}^{+0.063}$ \\
Sum rules [69,70] & 2 & $0.149_{-0.053}^{+0.052}$ & $0.06 \pm 0.10$ \\
LCSR fitting [72] & 1 & $0.17 \pm 0.08$ & \\
LCSR fitting [73] & 2 & 0.096 & $0.12-0.25$ \\
LCSR fitting [74] & 2 & 0.067 & 0.115 \\
LCSR fitting [71] & 1 & $0.22-0.33$ & 0.080 \\
Dyson-Schwinger (RL) [14] & 2 & 0.233 & \\
Dyson-Schwinger (DB) [14] & 2 & 0.149 & \\
\hline \hline
\end{tabular}



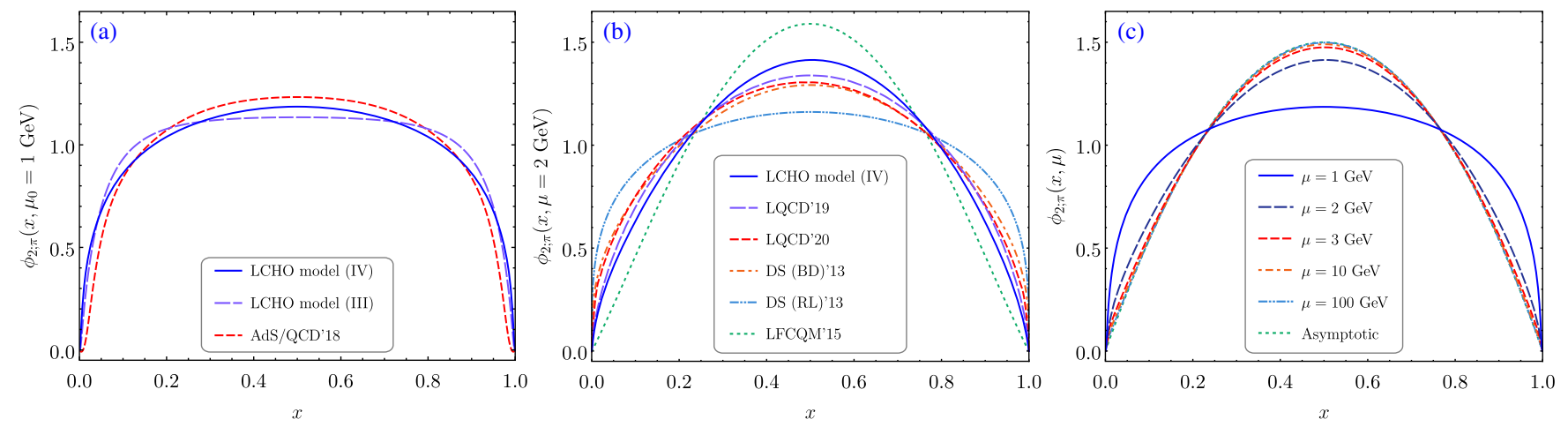

FIG. 3. Pionic leading-twist DA curves in this work. In panels (a) and (b) we present the DS model [14], QCD/AdS model [54], the DAs obtained using LFCQM [75], and LQCD [67,68] as a comparison. In panel (c) we show results from our LCHO model-IV at several typical energy scales, e.g., $\mu=1,2,3,10,100 \mathrm{GeV}$.

$$
\begin{aligned}
A_{2 ; \pi} & =14.7999 \mathrm{GeV}^{-1}, \\
\alpha_{2 ; \pi} & =-0.158, \\
\beta_{2 ; \pi} & =0.920029 \mathrm{GeV},
\end{aligned}
$$

with $\chi_{\min }^{2} / n_{d}=0.437236 / 4, \quad P_{\chi_{\min }^{2}}=0.979316 . \quad$ The parameters of our LCHO model-IV are

$$
\begin{aligned}
& A_{2 ; \pi}=5.95481 \mathrm{GeV}^{-1}, \\
& \alpha_{2 ; \pi}=-0.717, \\
& \hat{a}_{2}^{2 ; \pi}=-0.125, \\
& \beta_{2 ; \pi}=0.937482 \mathrm{GeV},
\end{aligned}
$$

with $\chi_{\min }^{2} / n_{d}=0.119251 / 3, P_{\chi_{\min }^{2}}=0.989431$.

The curves for our predictions are shown in Fig. 3. For comparison, results from the DS model [14], QCD/AdS model with $B=1$ [54], the DAs obtained using the lightfront constituent quark model (LFCQM) [75], and LQCD $[67,68]$ are also shown in Fig. 3.

(1) From Fig. 3(a), one can find that our LCHO modelIII is nearly flat in the region $x \in[0.2,0.8]$, and is a little wider than LCHO model-IV, both of which are very close to the AdS/QCD model. With the model parameters of LCHO model-IV in Eq. (40), one can calculate the moments of the DA, i.e., $\left.\left\langle\xi^{n}\right\rangle_{2 ; \pi}^{\mathrm{IV}}\right|_{\mu_{0}}=$ $(0.269,0.140,0.089,0.063,0.048)$ for $n=(2,4,6$, $8,10)$, respectively. These values are also very close to the reference results in Table III.

(2) By substituting our LCHO model-III with the parameters in Eq. (39) into Eq. (6), we can get $\left.\left\langle\xi^{n}\right\rangle_{2 ; \pi}^{\mathrm{III}}\right|_{\mu_{0}}=$ $(0.275,0.142,0.089,0.062,0.046)$ for $n=(2,4,6$, $8,10)$, respectively. Comparing our LCHO model-III with LCHO model-IV, the latter is better, which will be used in the following discussion and calculation, and we omit the label "IV."

(3) From Fig. 3(b), one can find that our LCHO model is narrower than the DS model, wider than the LFCQM, and closer to the LQCD result in Ref. [67].
The pionic twist-2 DA behavior of our model at any other scale can be related to that of an initial scale by using the energy evolution equation [41], which is shown in the Fig. 3(c). One can find the following.

(1) Our LCHO model at $\mu_{0}$ is significantly broader than the asymptotic form.

(2) With the increase of the scale $\mu$, our pionic leadingtwist DA model curve becomes narrower and closer to the asymptotic form. In particular, when the scale $\mu$ is lower than $2 \mathrm{GeV}$, our pionic leading-twist DA behavior is more sensitive to $\mu$, while when $\mu>2 \mathrm{GeV}$, it is close to the asymptotic behavior and insensitive to the scale $\mu$.

(3) In order to have a clear look at the changes of DA with factorization scale, one can set $x=0.5$ and the numerical results are $\phi_{2 ; \pi}^{\mathrm{IV}}(x=0.5, \mu)=(1.186$, $1.414,1.475,1.490,1.498)$ for $\quad \mu=(1,2,3,10$, 100) $\mathrm{GeV}$, respectively.

As a further step, the sensitivity/goodness of fit for the behavior of our LCHO model $\varphi_{2 ; \pi}^{\mathrm{IV}}(x)$ with the constituent quark mass, i.e., $m_{q}=(350,340, \ldots, 200) \mathrm{GeV}$ is also shown in Table $\mathrm{V}$, which indicates that the value of the goodness of fit increases as the constituent quark mass decreases. $P_{\chi_{\min }^{2}}$ is less than 0.9 when $m_{q}>300 \mathrm{MeV}$. In order to more intuitively understand the impact of $m_{q}$ on our $\phi_{2 ; \pi}(x, \mu)$, the curves of our LCHO model for the pionic leading-twist DA $\phi_{2 ; \pi}(x, \mu)$ at $\mu=1 \mathrm{GeV}$ with the constituent quark mass $m_{q}=(200,250,300,350) \mathrm{MeV}$ are shown in Fig. 4. One can find that as $m_{q}$ increases, our model tends to a flat-like form.

Within the resultant LCHO model of our predictions, there also exists a Jacobi factor $\sqrt{\partial k_{n} / \partial x}$ that contributes to the wave functions [76], which can be read off as

$$
\frac{\partial k_{n}}{\partial x}=\frac{M_{0}}{4 x \bar{x}}\left[1-\left(\frac{m_{q}^{2}-m_{\bar{q}}^{2}}{M_{0}^{2}}\right)^{2}\right],
$$

with $M_{0}^{2}=\left(\mathbf{k}_{\perp}^{2}+m_{q}^{2}\right) / x+\left(\mathbf{k}_{\perp}^{2}+m_{\bar{q}}^{2}\right) / \bar{x}$. Due to the invariant meson mass scheme [27-33], one can take 
TABLE V. Model parameters of our LCHO model with $\varphi_{2 ; \pi}^{\mathrm{IV}}(x)$ and the corresponding goodness of fit for several typical constituent quark mass values $m_{q}$.

\begin{tabular}{lccccc}
\hline \hline$m_{q}(\mathrm{MeV})$ & $A_{2 ; \pi}\left(\mathrm{GeV}^{-1}\right)$ & $\alpha_{2 ; \pi}$ & $B_{2}^{2 ; \pi}$ & $\beta_{2 ; \pi}(\mathrm{GeV})$ & $P_{\chi_{\min }^{2}}$ \\
\hline 350 & 2.24732 & -1.382 & -0.115 & 0.608317 & 0.797900 \\
340 & 2.40934 & -1.330 & -0.115 & 0.617249 & 0.824339 \\
330 & 2.55114 & -1.286 & -0.116 & 0.627146 & 0.848822 \\
320 & 2.70534 & -1.242 & -0.117 & 0.637809 & 0.871190 \\
310 & 2.92676 & -1.186 & -0.116 & 0.649553 & 0.891410 \\
300 & 3.10553 & -1.143 & -0.117 & 0.662378 & 0.909415 \\
290 & 3.32326 & -1.095 & -0.117 & 0.676766 & 0.925250 \\
280 & 3.52839 & -1.053 & -0.118 & 0.692543 & 0.938962 \\
270 & 3.82177 & -0.999 & -0.117 & 0.710313 & 0.950620 \\
260 & 4.14554 & -0.945 & -0.116 & 0.730185 & 0.960338 \\
250 & 4.45116 & -0.898 & -0.116 & 0.752879 & 0.968389 \\
240 & 4.79603 & -0.850 & -0.116 & 0.778565 & 0.974850 \\
230 & 5.21220 & -0.797 & -0.115 & 0.808690 & 0.979973 \\
220 & 5.63027 & -0.749 & -0.115 & 0.843751 & 0.983961 \\
210 & 5.85107 & -0.726 & -0.119 & 0.885935 & 0.987168 \\
200 & 5.95481 & -0.717 & -0.125 & 0.937482 & 0.989431 \\
\hline \hline
\end{tabular}

$m_{q}=m_{\bar{q}}$ for the pion cases, and thus the spatial wave function is

$\Psi_{2 ; \pi}^{R}\left(x, \mathbf{k}_{\perp}\right)=A_{2 ; \pi} \varphi_{2 ; \pi}(x) \frac{\sqrt{\mathbf{k}_{\perp}^{2}+m_{q}^{2}}}{4(x \bar{x})^{3 / 2}} \exp \left[-\frac{\mathbf{k}_{\perp}^{2}+m_{q}^{2}}{8 \beta_{2 ; \pi}^{2} x \bar{x}}\right]$.

Finally, we can get the expression for pionic twist-2 DA,

$$
\begin{aligned}
\phi_{2 ; \pi}(x, \mu)= & \frac{\sqrt{3 / 2} A_{2 ; \pi} m_{q} \beta_{2 ; \pi}^{2} \varphi_{2 ; \pi}(x)}{2 \pi^{2} f_{\pi} \sqrt{x \bar{x}}} \exp \left[-\frac{m_{q}^{2}}{8 \beta_{2 ; \pi}^{2} x \bar{x}}\right] \\
& \times\left\{1-\exp \left[-\frac{\mu^{2}}{8 \beta_{2 ; \pi}^{2} x \bar{x}}\right]\right\} .
\end{aligned}
$$

Then, we can fit the values of the moments $\left\langle\xi^{n}\right\rangle_{2 ; \pi}$ from the sum rules (11) by using the least squares method with the above model. Comparing the behavior of the two pionic leading-twist DA LCHO models with and without the Jacobi factor, the difference between the two is not obvious, as can be seen in Fig. 5 .

As significant applications, we recalculate the pionphoton TFF $F_{\pi \gamma}\left(Q^{2}\right)$ and the $B \rightarrow \pi$ TFF $f_{+}^{B \rightarrow \pi}\left(q^{2}\right)$ with our pionic leading-twist DA model. The pion-photon TFF $F_{\pi \gamma}\left(Q^{2}\right)$ can be calculated using $\operatorname{LCSR}[69,70,77]$ and the pQCD method $[19,78]$. With the pQCD method, $F_{\pi \gamma}\left(Q^{2}\right)$ can be expressed as the sum of the valence quark part contribution $F_{\pi \gamma}^{(\mathrm{V})}\left(Q^{2}\right)$ and the nonvalence quark part contribution $F_{\pi \gamma}^{(\mathrm{NV})}\left(Q^{2}\right)$,

$$
F_{\pi \gamma}\left(Q^{2}\right)=F_{\pi \gamma}^{(\mathrm{V})}\left(Q^{2}\right)+F_{\pi \gamma}^{(\mathrm{NV})}\left(Q^{2}\right)
$$

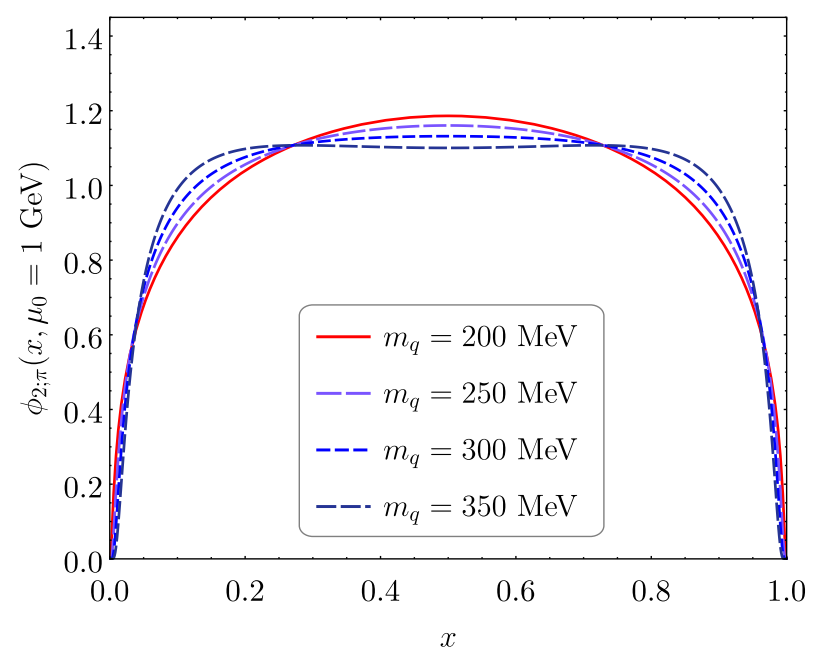

FIG. 4. Curves of our LCHO model for the pionic leading-twist DA $\phi_{2 ; \pi}(x, \mu)$ at $\mu=1 \mathrm{GeV}$ with the constituent quark mass $m_{q}=200,250,300,350 \mathrm{MeV}$, respectively.

where the corresponding analytical formulas for $F_{\pi \gamma}^{(\mathrm{V})}\left(Q^{2}\right)$ and $F_{\pi \gamma}^{(\mathrm{NV})}\left(Q^{2}\right)$ can be found in Refs. [19,78]. Figure 6 shows the curve of $Q^{2} F_{\pi \gamma}\left(Q^{2}\right)$ versus $Q^{2}$ obtained using our pionic leading-twist DA model and the experimental data reported by the CELLO [79], CLEO [80,81], BABAR [82], and Belle [83] collaborations, and one can find that our prediction is consistent with the BELLE data in the large- $Q^{2}$ region.

Furthermore, as another important application for the pion twist-2 DA, the TFF for the $B \rightarrow \pi$ decay processes should be analyzed. We start with the following correlation function:

$\Pi_{\mu}(p, q)=i \int d^{4} x e^{i q \cdot x}\left\langle\pi^{+}(p)\left|T\left\{j_{V}^{\mu}(x), j_{B}^{\dagger}(0)\right\}\right| 0\right\rangle$,

with $j_{V}^{\mu}(x)=\bar{u}(x) \gamma_{\mu}\left(1+\gamma_{5}\right) b(x)$. For the $B$-meson current $j_{B}^{\dagger}(0)$, we choose the right-handed current $j_{B}^{\dagger}(0)=$ $m_{b} b(0) i\left(1+\gamma_{5}\right) d(0)$ which can highlight the twist-2 and -4 DA contributions, and the twist-3 DAs contributions vanish. By following the standard procedures of the lightcone sum rules approach [84,85], we can get the $B \rightarrow \pi$ TFF $f_{+}^{B \rightarrow \pi}\left(q^{2}\right)$, which reads

$$
\begin{aligned}
f_{+}^{B \rightarrow \pi}\left(q^{2}\right)= & \frac{e^{m_{B}^{2} / M^{2}}}{m_{B}^{2} f_{B}}\left[F_{0}\left(q^{2}, M^{2}, s_{0}^{B}\right)\right. \\
& \left.+\frac{\alpha_{S} C_{F}}{4 \pi} F_{1}\left(q^{2}, M^{2}, s_{0}^{B}\right)\right],
\end{aligned}
$$

where $C_{F}=4 / 3, m_{B}$ and $f_{B}$ are the $B$-meson mass and decay constant, respectively, and $s_{0}^{B}$ is the continuum threshold. The LO contribution of the LCSR (46) is expressed as 

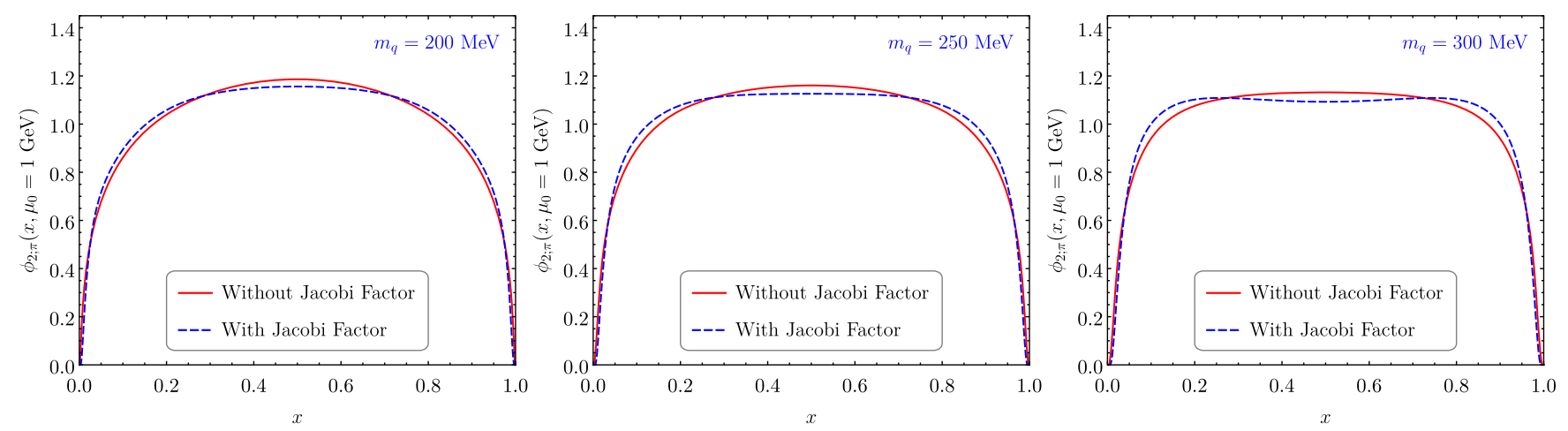

FIG. 5. Comparison of two pionic leading-twist DA LCHO models with and without the Jacobi factor. The three panels correspond to the constituent quark masses $m_{q}=200,250,300 \mathrm{MeV}$, respectively.

$$
\begin{aligned}
F_{0} & \left(q^{2}, M^{2}, s_{0}^{B}\right) \\
= & m_{b}^{2} f_{\pi} \int_{u_{0}}^{1} d u e^{-\frac{m_{b}^{2}-q^{2} \bar{u}}{u M^{2}}}\left\{\frac{\phi_{2 ; \pi}(u)}{u}+\frac{1}{m_{b}^{2}-q^{2}}\right. \\
& \times\left[-\frac{m_{b}^{2} u}{4\left(m_{b}^{2}-q^{2}\right)} \frac{d^{2} \phi_{4 \pi}(u)}{d u^{2}}+u \psi_{4 \pi}(u)\right. \\
& \left.\left.+\int_{0}^{u} d v \psi_{4 \pi}(v)-I_{4 \pi}(u)\right]\right\},
\end{aligned}
$$

and the NLO term of $f_{+}^{B \rightarrow \pi}\left(q^{2}\right)$ is

$$
\begin{aligned}
& F_{1}\left(q^{2}, M^{2}, s_{0}^{B}\right) \\
& =\frac{f_{\pi}}{\pi} \int_{m_{b}^{2}}^{s_{0}^{B}} d s e^{-s / M^{2}} \int_{0}^{1} d u \operatorname{Im}_{s} T_{1}\left(q^{2}, s, u\right) \phi_{2 ; \pi}(u),
\end{aligned}
$$

where $m_{b}$ is the $b$-quark mass, $\bar{u}=1-u, u_{0}=\left(m_{b}^{2}-q^{2}\right) /$ $\left(s_{0}^{B}-q^{2}\right), \phi_{4 \pi}(u)$ and $\psi_{4 \pi}(u)$ are the pionic twist-4 DAs, and $I_{4 \pi}(u)$ is the combined function of the four pionic

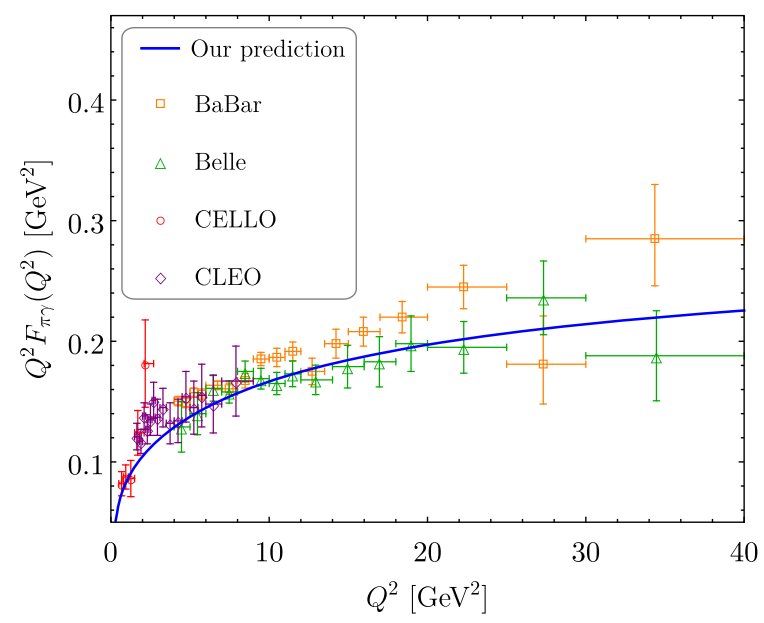

FIG. 6. Pion-photon TFF $Q^{2} F_{\pi \gamma}^{(V)}\left(Q^{2}\right)$ with our model. For comparison, the experimental data reported by the CELLO [79], CLEO [80,81], BABAR [82], and Belle [83] collaborations are shown. twist-4 DAs $\Psi_{4 \pi}(u), \Phi_{4 \pi}(u), \tilde{\Psi}_{4 \pi}(u)$, and $\tilde{\Phi}_{4 \pi}(u)$. One can find the expressions for these pionic twist- 4 DAs, $I_{4 \pi}(u)$, and the imaginary part of the amplitude $T_{1}$ in Ref. [85]. By taking $\mu=3 \mathrm{GeV}, M^{2}=18 \pm 1 \mathrm{GeV}^{2}, s_{0}^{B}=35.75 \pm$ $0.25 \mathrm{GeV}^{2}, m_{B}=5.279 \mathrm{GeV}$, and $f_{B}=214_{-5}^{+7} \mathrm{MeV}$ [85], we can obtain

$$
f_{+}^{B \rightarrow \pi}(0)=0.295_{-0.013}^{+0.018} \text {. }
$$

This value is consistent with that of other theoretical group [85-88] by the conventional current correlation. The difference between the central value in Eq. (49) and that in Ref. [85] is mainly due to the difference in the selected correlation function. Comparing Eqs. (46)-(48) above with Eqs. (4.4), (4.5), and (4.7) in Ref. [85], one can find that the contributions from the pionic twist-3 DAs disappear, while the contributions from the pionic twist- 2 and -4 DAs are doubled. Then, the difference between the twist-2 DA's contribution and twist-3 DAs' contributions in the LCSR with the conventional current correlation can be used as the systematic error caused by adopting the chiral current correlation function.

\section{SUMMARY}

In this paper, we have improved the traditional LCHO model of the pionic leading-twist DA $\phi_{2 ; \pi}(x, \mu)$ by introducing a new WF's longitudinal DA, i.e., $\varphi_{2 ; \pi}^{\mathrm{IV}}$ in Eq. (21). At the same time, we have improved the method of determining the model parameters. More explicitly, the least squares method was adopted to fit the moments $\left.\left\langle\xi^{n}\right\rangle_{2 ; \pi}\right|_{\mu}$ directly to determine the model parameters. This makes it necessary and meaningful to calculate higher-order moments, and we can obtain a stronger constraint on the DA behavior by including more moments.

We have adopted the QCD sum rules based on the BFT to calculate the moments $\left.\left\langle\xi^{n}\right\rangle_{2 ; \pi}\right|_{\mu}$, and the values of the first five moments are $\left.\left\langle\xi^{2}\right\rangle_{2 ; \pi}\right|_{\mu_{0}}=0.271 \pm 0.013$, $\left.\left\langle\xi^{4}\right\rangle_{2 ; \pi}\right|_{\mu_{0}}=0.138 \pm 0.010,\left.\quad\left\langle\xi^{6}\right\rangle_{2 ; \pi}\right|_{\mu_{0}}=0.087 \pm 0.008$, 
$\left.\left\langle\xi^{8}\right\rangle_{2 ; \pi}\right|_{\mu_{0}}=0.064 \pm 0.007, \quad$ and $\left.\quad\left\langle\xi^{10}\right\rangle_{2 ; \pi}\right|_{\mu_{0}}=0.050 \pm$ 0.006 . Based on these values, we obtained the behavior of $\phi_{2 ; \pi}(x, \mu)$, that is, Eqs. (15), (21), and (40).

Compared with our previous work, in addition to the improvement of the LCHO model, there are three improvements: (i) the moments $\left.\left\langle\xi^{n}\right\rangle_{2 ; \pi}\right|_{\mu}$, rather than the Gegenbauer moments $a_{n}^{2 ; \pi}(\mu)$, were used as constraint conditions to determine the model parameters; (ii) the least squares method was used to fit the moments $\left.\left\langle\xi^{n}\right\rangle_{2 ; \pi}\right|_{\mu}$ to get the appropriate model parameters; (iii) we took Eq. (11) rather than Eq. (7) as the sum rules of $\left.\left\langle\xi^{n}\right\rangle_{2 ; \pi}\right|_{\mu}$, which can avoid the error caused by the non-normalized zeroth moment $\left.\left\langle\xi^{0}\right\rangle_{2 ; \pi}\right|_{\mu}$ on the left-hand side of Eq. (7), and cause the accuracy of the resulting values of $\left.\left\langle\xi^{n}\right\rangle_{2 ; \pi}\right|_{\mu}$ to increase by more than $10 \%$. These improvements can be widely used in QCD sum rules studies of other meson DAs to obtain more accurate DA behavior.

As an application, we have used our model to calculate the pion-photon TFF $F_{\pi \gamma}\left(Q^{2}\right)$, which is shown in Fig. 6.
Our results agree with the Belle predictions in the large $Q^{2}$-region. Meanwhile, the $B \rightarrow \pi$ TFF $f_{+}^{B \rightarrow \pi}\left(q^{2}\right)$ has been calculated up to NLO accuracy, which agrees with other theoretical predictions.

\section{ACKNOWLEDGMENTS}

This work was supported in part by the National Natural Science Foundation of China under Grants No. 11765007, No. 11875122, No. 11625520 , No. 11947406, and No. 12047564, the Project of Guizhou Provincial Department of Science and Technology under Grants No. KY[2019]1171 and No. ZK[2021]024, the Project of Guizhou Provincial Department of Education under Grant No. KY[2021]030, the Chongqing Graduate Research and Innovation Foundation under Grant No. ydstd1912, the Fundamental Research Funds for the Central Universities under Grant No. 2020CQJQY-Z003, and the Project of Guizhou Minzu University under Grant No. GZMU[2019]YB19.
[1] S. J. Brodsky and G. P. Lepage, in: Perturbative Quantum Chromodynamics, ed. by A.H. Mueller, p. 93, World Scientific (Singapore) 1989.

[2] G. P. Lepage and S. J. Brodsky, Exclusive Processes in Quantum Chromodynamics: Evolution Equations for Hadronic Wave Functions and the Form-Factors of Mesons, Phys. Lett. B 87 (1979) 359.

[3] A. V. Efremov and A. V. Radyushkin, Factorization and Asymptotical Behavior of Pion Form-Factor in QCD, Phys. Lett. B 94 (1980) 245.

[4] V. L. Chernyak and A. R. Zhitnitsky, Exclusive Decays of Heavy Mesons, Nucl. Phys. B201 (1982) 492.

[5] V. L. Chernyak and A. R. Zhitnitsky, Asymptotic Behavior of Exclusive Processes in QCD, Phys. Rept. 112 (1984) 173.

[6] V. L. Chernyak and I. R. Zhitnitsky, Nucleon Wave Function and Nucleon Form-Factors in QCD, Nucl. Phys. B246 (1984) 52.

[7] T. Huang, X. N. Wang, and X. D. Xiang, The distribution amplitude for pion and QCD sum rules, Chin. Phys. Lett. 2 (1985) 67.

[8] X. D. Xiang, X. N. Wang, and T. Huang, QCD sum rule and meson distribution amplitude moments, Commun. Theor. Phys. 6 (1986) 117.

[9] T. Huang, X. N. Wang, X. D. Xiang, and S. J. Brodsky, The quark mass and spin effects in the mesonic structure, Phys. Rev. D 35 (1987) 1013.

[10] S. A. Gottlieb and A. S. Kronfeld, Experimental predictions of lattice and perturbative Quantum Chromodynamics, Phys. Rev. Lett. 55 (1985) 2531.

[11] S. A. Gottlieb and A. S. Kronfeld, Lattice and perturbative QCD analysis of exclusive processes, Phys. Rev. D 33 (1986) 227.
[12] G. Martinelli and C. T. Sachrajda, The quark distribution amplitude of the proton: A lattice computation of the lowest two moments, Phys. Lett. B 217 (1989) 319.

[13] D. Daniel, R. Gupta, and D. G. Richards, A Calculation of the pion's quark distribution amplitude in lattice QCD with dynamical fermions, Phys. Rev. D 43, 3715 (1991).

[14] L. Chang, I. C. Cloet, J. J. Cobos-Martinez, C. D. Roberts, S. M. Schmidt, and P. C. Tandy, Imaging dynamical chiral symmetry breaking: pion wave function on the light front, Phys. Rev. Lett. 110 (2013) 132001. [arXiv:1301.0324]

[15] S. J. Brodsky, T. Huang, and G. P. Lepage, in Particles and Fields-2, Proceedings of the Banff Summer Institute, Ban8; Alberta1981, edited by A.Z. Capri and A. N. Kamal (Plenum, New York, 1983), p. 143; G. P. Lepage, S. J. Brodsky, T. Huang, and P. B. Mackenize, ibid., p. 83; T. Huang, in Proceedings of XXth International Conference on High Energy Physics, Madison, Wisconsin, 1980, edited by L. Durand and L. G Pondrom, AIP Conf. Proc. No. 69 (AIP, New York, 1981), p. 1000.

[16] M. Ahmady, F. Chishtie, and R. Sandapen, The pion in holographic light-front QCD, Nucl. Part. Phys. Proc. 294 (2018) 119. [arXiv:1709.01125].

[17] T. Huang and Z. Huang, Quantum Chromodynamics in Background Fields, Phys. Rev. D 39 (1989) 1213.

[18] T. Zhong, X. G. Wu, Z. G. Wang, T. Huang, H. B. Fu, and H. Y. Han, Revisiting the pion leading-twist distribution amplitude within the QCD background field theory, Phys. Rev. D 90 (2014) 016004. [arXiv:1405.0774].

[19] X. G. Wu and T. Huang, An implication on the pion distribution amplitude from the pion-photon transition form factor with the new BABAR data, Phys. Rev. D 82 (2010) 034024. [arXiv:1005.3359]. 
[20] X. G. Wu and T. Huang, Constraints on the light pseudoscalar meson distribution amplitudes from their mesonphoton transition form factors, Phys. Rev. D 84 (2011) 074011. [arXiv:1106.4365].

[21] T. Huang, B. Q. Ma, and Q. X. Shen, Analysis of the pion wave function in light cone formalism, Phys. Rev. D 49 (1994) 1490. [arXiv:hep-ph/9402285]

[22] F. G. Cao and T. Huang, Large corrections to asymptotic $F\left(\eta_{c} \gamma\right)$ and $F\left(\eta_{b} \gamma\right)$ in the light cone perturbative QCD, Phys. Rev. D 59 (1999) 093004. [arXiv:hep-ph/9711284].

[23] T. Huang and X. G. Wu, A Model for the twist-3 wave function of the pion and its contribution to the pion formfactor, Phys. Rev. D 70 (2004) 093013. [arXiv:hep-ph/ 0408252]

[24] X. G. Wu and T. Huang, Pion electromagnetic form-factor in the $\mathrm{K}(\mathrm{T})$ factorization formulae, Int. J. Mod. Phys. A 21 (2006) 901. [arXiv:hep-ph/0507136]

[25] See, e.g., Elementary Particle Theory Group, Acta Phys. Sin. 25, 415 (1976); N. Isgur, in The New Aspects ofSubnu clear Physics, edited by A. Zichichi (Plenum, New York, 1980), p. 107.

[26] T. Zhong, X. G. Wu, and T. Huang, The longitudinal and transverse distributions of the pion wave function from the present experimental data on the pion-photon transition form factor, Eur. Phys. J. C 76 (2016) 390. [arXiv:1510.06924]

[27] M. V. Terentev, On the Structure of Wave Functions of Mesons as Bound States of Relativistic Quarks, Sov. J. Nucl. Phys. 24 (1976) 106 ITEP-5-1976, https://inspirehep.net/ literature/109844.

[28] W. Jaus, Semileptonic Decays of $B$ and $D$ Mesons in the Light Front Formalism, Phys. Rev. D 41 (1990) 3394.

[29] W. Jaus, Relativistic constituent quark model of electroweak properties of light mesons, Phys. Rev. D 44 (1991) 2851.

[30] P. L. Chung, F. Coester, and W. N. Polyzou, Charge FormFactors of Quark Model Pions, Phys. Lett. B 205 (1988) 545.

[31] H. M. Choi and C. R. Ji, Relations among the light cone quark models with the invariant meson mass scheme and the model prediction of $\eta-\eta^{\prime}$ mixing angle, Phys. Rev. D 56 (1997) 6010.

[32] F. Schlumpf, Charge form-factors of pseudoscalar mesons, Phys. Rev. D 50 (1994) 6895. [arXiv:hep-ph/9406267]

[33] F. Cardarelli, I. L. Grach, I. M. Narodetsky, G. Salme, and S. Simula, Electromagnetic form-factors of the $\rho$ meson in a light front constituent quark model, Phys. Lett. B 349 (1995) 393. [arXiv:hep-ph/9502360]

[34] Z. Dziembowski and L. Mankiewicz, textitLight Meson Distribution Amplitude: A Simple Relativistic Model, Phys. Rev. Lett. 58 (1987) 2175.

[35] Z. Dziembowski, Relativistic Model of Nucleon and Pion Structure: Static Properties and Electromagnetic Soft Formfactors, Phys. Rev. D 37 (1988) 778.

[36] C. R. Ji and S. R. Cotanch, Simple relativistic quark model analysis of flavored pseudoscalar mesons, Phys. Rev. D 41 (1990) 2319.

[37] C. R. Ji, P. L. Chung, and S. R. Cotanch, Light cone quark model axial vector meson wave function, Phys. Rev. D 45 (1992) 4214.

[38] H. M. Choi and C. R. Ji, Light cone quark model predictions for radiative meson decays, Nucl. Phys. A618 (1997) 291.
[39] X. G. Wu, T. Huang, and T. Zhong, Information on the pion distribution amplitude from the pion-photon transition form factor with the Belle and BABAR data, Chin. Phys. C 37 (2013) 063105. [arXiv:1206.0466]

[40] T. Huang, X. G. Wu, and T. Zhong, Finding a way to determine the pion distribution amplitude from the experimental data, Chin. Phys. Lett. 30 (2013) 041201. [arXiv:1303.2301]

[41] T. Huang, T. Zhong, and X. G. Wu, Determination of the pion distribution amplitude, Phys. Rev. D 88 (2013) 034013. [arXiv:1305.7391]

[42] T. Zhong, X. G. Wu, and T. Huang, Heavy Pseudoscalar Leading-Twist Distribution Amplitudes within QCD Theory in Background Fields, Eur. Phys. J. C 75 (2015) 45. [arXiv:1408.2297]

[43] T. Zhong, X. G. Wu, T. Huang, and H. B. Fu, Heavy pseudoscalar twist-3 distribution amplitudes within QCD theory in background fields, Eur. Phys. J. C 76 (2016) 509. [arXiv:1604.04709]

[44] Y. Zhang, T. Zhong, X. G. Wu, K. Li, H. B. Fu, and T. Huang, Uncertainties of the $B \rightarrow D$ transition form factor from the D-meson leading-twist distribution amplitude, Eur. Phys. J. C 78 (2018) 76. [arXiv:1709.02226]

[45] T. Zhong, Y. Zhang, X. G. Wu, H. B. Fu, and T. Huang, The ratio $\mathcal{R}(D)$ and the $D$-meson distribution amplitude, Eur. Phys. J. C 78 (2018) 937. [arXiv:1807.03453]

[46] P. A. Zyla et al. (Particle Data Group), Review of Particle Physics, Prog. Theor. Exp. Phys. 2020 (2020) $083 \mathrm{C} 01$.

[47] S. Narison, Improved $f_{D^{*}}, f_{B^{*}}$ and $f_{B_{c}}$ from QCD Laplace sum rules, Int. J. Mod. Phys. A 30 (2015) 1550116. [arXiv:1404.6642]

[48] P. Colangelo and A. Khodjamirian, QCD sum rules, a modern perspective, arXiv:hep-ph/0010175.

[49] S. Narison, Mini-review on QCD spectral sum rules, Nucl. Part. Phys. Proc. 258-259 (2015) 189. [arXiv:1409.8148]

[50] K. C. Yang, W. Y. P. Hwang, E. M. Henley, and L. S. Kisslinger, QCD sum rules and neutron proton mass difference, Phys. Rev. D 47 (1993) 3001.

[51] W. Y. P. Hwang and K. C. Yang, QCD sum rules: $\Delta-N$ and $\Sigma^{0}-\Lambda$ mass splittings, Phys. Rev. D 49 (1994) 460.

[52] C. D. Lü, Y. M. Wang, and H. Zou, Twist-3 distribution amplitudes of scalar mesons from QCD sum rules, Phys. Rev. D 75 (2007) 056001. [arXiv:hep-ph/0612210]

[53] S. X. Qin and C. D. Roberts, Impressions of the continuum bound state problem in QCD, Chin. Phys. Lett. 37 (2020) 121201. [arXiv:2008.07629]

[54] M. Ahmady, C. Mondal, and R. Sandapen, Dynamical spin effects in the holographic light-front wavefunctions of light pseudoscalar mesons, Phys. Rev. D 98 (2018) 034010. [arXiv:1805.08911]

[55] S. J. Brodsky and G. F. de Teramond, Light-Front dynamics and AdS/QCD correspondence: the pion form factor in the space- and time-Like regions, Phys. Rev. D 77 (2008) 056007. [arXiv:0707.3859]

[56] N. G. Stefanis, What binds quarks together at different momentum scales? A conceptual scenario, Phys. Lett. B 738 (2014) 483-487. [arXiv:1405.0959]

[57] H. M. Choi and C. R. Ji, Distribution amplitudes and decay constants for $\left(\pi, K, \rho, K^{*}\right)$ mesons in light-front quark 
model, Phys. Rev. D 75 (2007) 034019. [arXiv:hep-ph/ 0701177]

[58] P. Ball and R. Zwicky, New results on $B \rightarrow \pi, K, \eta$ decay formfactors from light-cone sum rules, Phys. Rev. D 71 (2005) 014015. [arXiv:hep-ph/0406232]

[59] S. S. Agaev, Impact of the higher twist effects on the $\gamma \gamma^{*} \rightarrow \pi^{0}$ transition form-factor, Phys. Rev. D 72 (2005) 114010. [arXiv:hep-ph/0511192]

[60] V. Y. Petrov, M. V. Polyakov, R. Ruskov, C. Weiss, and K. Goeke, Pion and photon light cone wave functions from the instanton vacuum, Phys. Rev. D 59 (1999) 114018. [arXiv: hep-ph/9807229]

[61] S. i. Nam, H. C. Kim, A. Hosaka, and M. M. Musakhanov, The Leading-twist pion and kaon distribution amplitudes from the QCD instanton vacuum, Phys. Rev. D 74 (2006) 014019. [arXiv:hep-ph/0605259]

[62] A. P. Bakulev, S. V. Mikhailov, and N. G. Stefanis, QCD based pion distribution amplitudes confronting experimental data, Phys. Lett. B 508 (2001) 279. [arXiv:hep$\mathrm{ph} / 0103119]$

[63] R. Arthur, P. A. Boyle, D. Brommel, M. A. Donnellan, J. M. Flynn, A. Juttner, T. D. Rae, and C. T. C. Sachrajda, Lattice results for low moments of light meson distribution amplitudes, Phys. Rev. D 83 (2011) 074505. [arXiv:1011.5906]

[64] V. M. Braun, S. Collins, M. Göckeler, P. Pérez-Rubio, A. Schäfer, R. W. Schiel, and A. Sternbeck, Second moment of the pion light-cone distribution amplitude from lattice QCD, Phys. Rev. D 92 (2015) 014504. [arXiv:1503.03656]

[65] V. M. Braun, M. Gockeler, R. Horsley, H. Perlt, D. Pleiter, P. E. L. Rakow, G. Schierholz, A. Schiller, W. Schroers and H. Stuben et al., Moments of pseudoscalar meson distribution amplitudes from the lattice, Phys. Rev. D 74, 074501 (2006). [arXiv:hep-lat/0606012]

[66] G. S. Bali et al. (RQCD Collaboration), Second moment of the pion distribution amplitude with the momentum smearing technique, Phys. Lett. B 774 (2017) 91. [arXiv:1705.10236]

[67] G. S. Bali et al. (RQCD Collaboration), Light-cone distribution amplitudes of pseudoscalar mesons from lattice QCD, J. High Energy Phys. 1908 (2019) 065.[2011, 037 (A) (2020)] [arXiv:1903.08038]

[68] R. Zhang, C. Honkala, H. W. Lin, and J. W. Chen, Pion and kaon distribution amplitudes in the continuum limit, Phys. Rev. D 102 (2020) 094519. [arXiv:2005.13955]

[69] S. V. Mikhailov, A. V. Pimikov, and N. G. Stefanis, Systematic estimation of theoretical uncertainties in the calculation of the pion-photon transition form factor using light-cone sum rules, Phys. Rev. D 93 (2016) 114018. [arXiv:1604.06391]

[70] N. G. Stefanis, Pion-photon transition form factor in light cone sum rules and tests of asymptotics, Phys. Rev. D 102 (2020) 034022. [arXiv:2006.10576]

[71] R. Bruschini and P. González, Diabatic description of charmoniumlike mesons, Phys. Rev. D 102 (2020) 074002. [arXiv:2007.07693]

[72] A. Khodjamirian, T. Mannel, N. Offen, and Y.-M. Wang, $B \rightarrow \pi \ell \nu_{l}$ width and $\left|V_{u b}\right|$ from QCD light-cone sum rules, Phys. Rev. D 83 (2011) 094031. [arXiv:1103.2655]
[73] S. S. Agaev, V. M. Braun, N. Offen, and F. A. Porkert, Light Cone Sum Rules for the $\pi^{0} \gamma^{*} \gamma$ Form Factor Revisited, Phys. Rev. D 83 (2011) 054020, [arXiv:1012.4671].

[74] S. S. Agaev, V. M. Braun, N. Offen, and F. A. Porkert, BELLE Data on the $\pi^{0} \gamma^{*} \gamma$ Form Factor: A Game Changer?, Phys. Rev. D 86 (2012) 077504. [arXiv:1206.3968]

[75] J. P. B. C. de Melo, I. Ahmed, and K. Tsushima, Parton Distribution in Pseudoscalar Mesons with a Light-Front Constituent Quark Model, AIP Conf. Proc. 1735 (2016) 080012. [arXiv:1512.07260]

[76] H. M. Choi and C. R. Ji, Mixing angles and electromagnetic properties of ground state pseudoscalar and vector meson nonets in the light cone quark model, Phys. Rev. D 59 (1999) 074015. [arXiv:hep-ph/9711450]

[77] S. V. Mikhailov, A. V. Pimikov, and N. G. Stefanis, Extending the application of the light-cone sum rules method to low momenta using QCD renormalization-group summation: Theory and phenomenology, Phys. Rev. D 103 (2021) 096003, [arXiv:2101.12661].

[78] T. Huang and X. G. Wu, A comprehensive analysis on the pion-photon transition form factor involving the transverse momentum corrections, Int. J. Mod. Phys. A 22 (2007) 3065. [arXiv:hep-ph/0606135]

[79] H. J. Behrend et al. (CELLO Collaboration), A Measurement of the $\pi^{0}, \eta$ and $\eta^{\prime}$ electromagnetic form-factors, Z. Phys. C 49 (1991) 401.

[80] V. Savinov et al. (CLEO Collaboration), Measurements of the meson-photon transition form factors of light pseudoscalar mesons at large momentum transfer, arXiv:hep-ex/ 9707028.

[81] J. Gronberg et al. (CLEO Collaboration), Measurements of the meson-photon transition form-factors of light pseudoscalar mesons at large momentum transfer, Phys. Rev. D 57 (1998) 33. [arXiv:hep-ex/9707031]

[82] B. Aubert et al. [BABAR Collaboration], Measurement of the $\gamma \gamma^{*} \rightarrow \pi^{0}$ transition form factor, Phys. Rev. D 80 (2009) 052002. [arXiv:0905.4778]

[83] S. Uehara et al. (Belle Collaboration), Measurement of $\gamma \gamma^{*} \rightarrow \pi^{0}$ transition form factor at Belle, Phys. Rev. D 86 (2012) 092007. [arXiv:1205.3249]

[84] T. Huang, Z. H. Li, and X. Y. Wu, Improved approach to the heavy to light form-factors in the light cone QCD sum rules, Phys. Rev. D 63 (2001) 094001.

[85] G. Duplancic, A. Khodjamirian, T. Mannel, B. Melic, and N. Offen, Light-cone sum rules for $B \rightarrow \pi$ form factors revisited, JHEP 0804 (2008) 014. [arXiv:0801.1796]

[86] Z. H. Li, N. Zhu, X. J. Fan, and T. Huang, Form Factors $f_{+}^{B \rightarrow \pi}(0)$ and $f_{+}^{D \rightarrow \pi}(0)$ in $Q C D$ and Determination of $\left|V_{u b}\right|$ and $\left|V_{c d}\right|$, JHEP 1205 (2012) 160. [arXiv:1206.0091]

[87] I. Sentitemsu Imsong, A. Khodjamirian, T. Mannel, and D. van Dyk, Extrapolation and unitarity bounds for the $B \rightarrow \pi$ form factor, JHEP 1502 (2015) 126. [arXiv:1409 .7816]

[88] A. Khodjamirian and A. V. Rusov, $B_{s} \rightarrow K \ell \nu_{\ell}$ and $B_{(s)} \rightarrow$ $\pi(K) \ell^{+} \ell^{-}$decays at large recoil and CKM matrix elements, JHEP 1708 (2017) 112. [arXiv:1703.04765] 